\title{
Mitteilungen der DGN
}

Nervenarzt 2020 • 91:969-988

https://doi.org/10.1007/s00115-020-01005-9

○) Springer Medizin Verlag GmbH, ein Teil von Springer Nature 2020

\section{Redaktion}

Prof. Dr. Gereon Nelles, Schriftführer, nelles@dgn.org (v.i.S.d.P.)

Neuromed-Campus Hohenlind, Werthmannstr. 1c, 50935 Köln

Prof. Dr. med. Peter Berlit, Generalsekretär, berlit@dgn.org

Dr. phil. Bettina Albers, Dr. med. Martina Berthold

Prof. Dr. Christine Klein, Präsidentin, klein@dgn.org

Dr. rer. nat. Thomas Thiekötter, Geschäftsführer, thiekoetter@dgn.org

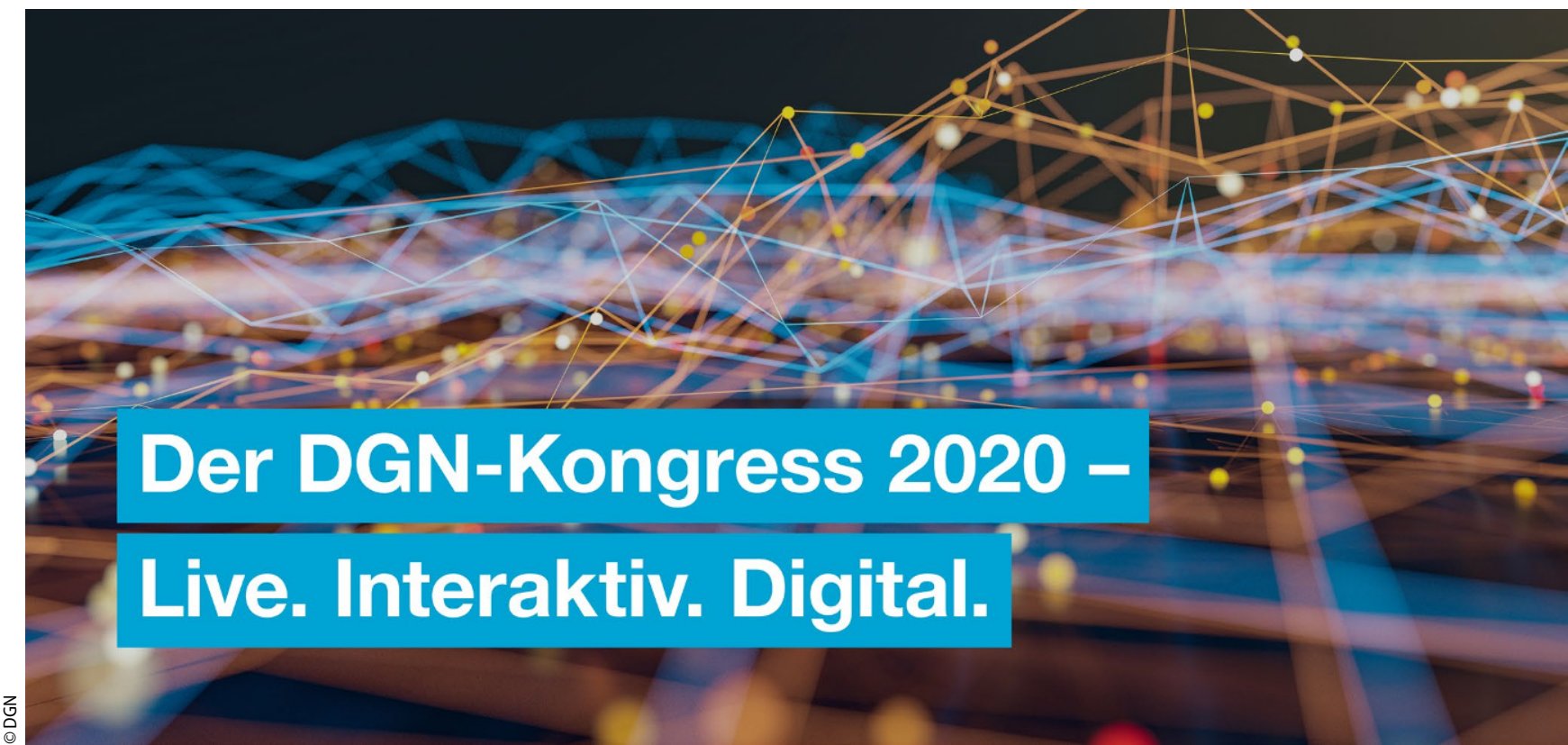

\section{DGN-Kongress im digitalen Exil}

Freuen Sie sich mit uns auf den DGN-Kongress $2020 \mathrm{im}$ „digitalen Exil"! Es erwartet Sie ein innovatives Veranstaltungsformat: Denn wir verstehen unter einem virtuellen Kongress mehr als nur das Ablegen von Präsentationen auf einer Online-Plattform. Wir werden die digitalen Möglichkeiten voll ausschöpfen, um das, was unseren Kongress ausmacht - die Interaktivität, die Diskussionen, das „Wirsind Neurologie-Gefühl" und das "Live“-Erlebnis -, auch im „CoronaExil" umzusetzen.

\section{Live, interaktiv und digital - was genau erwartet die Kongressbesucher?}

\section{Das Live-Programm}

Der DGN-Kongress bietet an allen Kongresstagen ein Live-Vortragsprogramm auf drei (am Freitag sogar auf vier) parallelen Kanälen an, die jeder Teilnehmer zu Hause oder am Arbeitsplatz über ein browserbasiertes Portal am Rechner mitverfolgen und zwischen denen er auch hinund herschalten kann. Für diese Live-Programme werden Studios in Berlin angemietet und die Referentinnen und Referenten werden dort im bekannten DGNKongressambiente ihre Vorträge halten und Diskussionen führen, die in Echtzeit gestreamt werden.
Diese Live-Veranstaltungen machen das Quäntchen mehr aus: Sie transportieren das eigentliche Kongresserlebnis, die Online-Teilnehmer sehen die "große Bühne" so, als wären sie im Kongresssaal, die Technik erlaubt außerdem echte Interaktion, Teilnehmer können ihre Fragen stellen und mitdiskutieren.

Das Live-Programm läuft nachmittags und abends, sodass es für viele Teilnehmer möglich sein wird, trotz Klinik- und Praxisbetrieb teilzunehmen. Für alle, die es nicht einrichten können: Registrierte Kongressbesucher können die Sessions des LiveProgramms ab dem nächsten Tag als Webcasts abrufen.

\section{Außerdem: das Webcast- \\ Programm}

Neben den Live-Kanälen, die auch im Nachgang angeschaut werden können, gibt es viele zusätzliche Kongressinhalte, die auf der Kon- gressplattform hinterlegt werden - natürlich ebenfalls hochkarätig produziert und immer auch mit professioneller Videoaufnahme des Sprechers: Denn neuronale Stimulation und Vernetzung ist unser Kerngeschäft!

Die Vorträge von ca. 300 Referentinnen und Referenten werden professionell vorproduziert. Diese Vorträge machen den Großteil des Kongressprogramms aus, das im Umfang kaum dem eines Präsenzkongresses nachsteht. Diese Vorträge stehen registrierten Kongressteilnehmern ab dem ersten Kongresstag - sortiert nach Themen und Fachbereichen - ein Jahr lang auf dem Kongressportal zur Verfügung.

Kongressteilnehmer haben also ein Jahr lang Zugriff auf alle Kongressinhalte inklusive der bereits bewährten E-LearningFormate. Das angebotene Online-Kongressprogramm ist nahezu so umfangreich wie das der 
vergangenen Präsenzkongresse und kann nach Themen und Redass die Nutzer die gewünschten Inhalte schnell finden.

Für den virtuellen DGN-Kongress werden CME-Punkte bei der Ärztekammer Berlin beantragt. Zusätzliche CME-Punkte können wie gewohnt über unser E-Learning-Angebot generiert werden, welches ebenfalls ab 4. November 2020 ein Jahr lang online zur Verfügung steht.

\section{Alles für einen Preis!}

Alle Vorträge können 24 Stunden an 7 Tagen pro Woche und 365 Tagen im Jahr von allen registrierten Kongressteilnehmern angeschaut werden. Voraussetzung ist ein internetfähiges Endgerät (PC, Laptop, Smartphone oder Tablet). Die Videoforen stehen exklusiv nur den Live-Zuschauern zur Verfügung. Das Live-Feeling am 4., 5., 6. und 7. November 2020 sollten Sie sich auch aus diesem Grund nicht entgehen lassen!

Die Kongressregistrierung umfasst das komplette 24/7/365-Paket: ein großes Angebot zu einem fairen Preis!

Programm und Anmeldung online unter: https://www.dgnkongress.org ferenten durchsucht werden, so-

\section{Das Live-Kongress- programm: parallel auf mehreren Kanälen}

\section{Wissenschaft Live}

Auf diesem Kanal werden die wissenschaftlichen Sitzungen ausgestrahlt, allen voran die Eröffnungsveranstaltung und das Präsidentensymposium. Darüber hinaus gibt es Sitzungen zu den Themen „COVID-19 in der Neurologie“, ,Schlaganfall“, ,Neuroimmunologie“, „Demenz", weitere Live-Sessions sind „Highlights der klinischen Neurologie: Neurology in Progress", ein beliebtes Format, in dem Prof. Dr. Hans-Christoph Diener die wichtigsten klinischen Studien in der Neurologie vorstellt und erklärt, wie sich die Ergebnisse der Studien in der klinischen Praxis auswirken, „Neurologie und Neurologen im Nationalsozialismus“ und „Spotlight: Höhepunkte des Kongresses“.

Außerdem werden im LiveKongressstrang vier Videoforen präsentiert: Dieses Veranstaltungsformat ist weit mehr als nur eine mediale, dem Zeitgeist geschuldete Spielerei. Es hat sich durchgesetzt, weil gerade in der Neurologie die phänomenologische Befundung eine große Rolle spielt und Videokasuistiken einen großen Lehrgehalt haben. Videoforen sind damit innovative Formate der Weiterbildung.

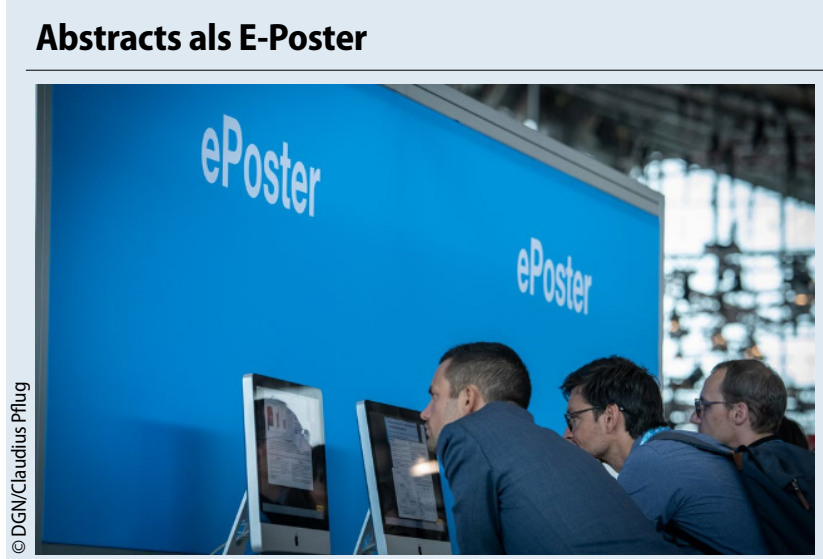

Alle akzeptierten Abstracts werden en bloc ab 4. November 2020 digital veröffentlicht, und zwar in Form eines E-Posters. Die Einreichenden haben die Möglichkeit, zusätzlich zu ihren E-Postern einen Audiokommentar abzugeben, welcher zum Poster online gestellt wird.

\section{Jetzt aber noch schnell anmelden!}

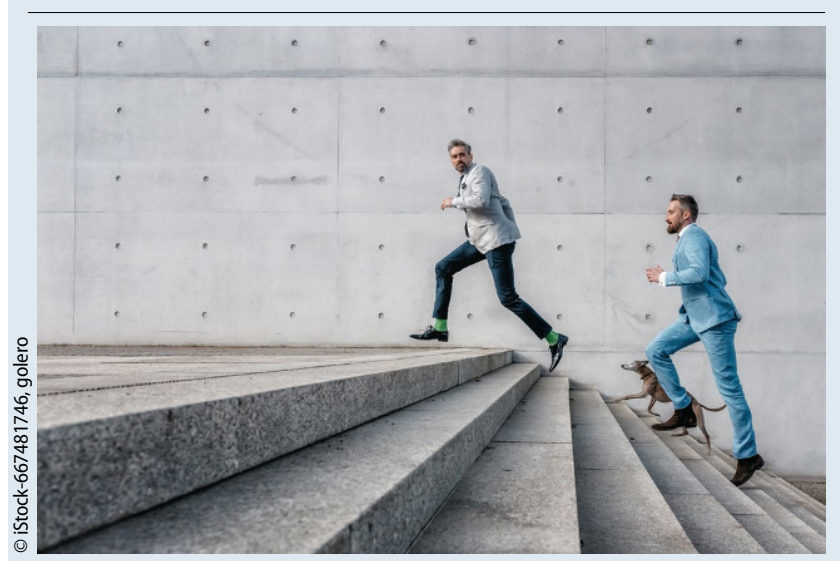

Lust bekommen? Dann halten Sie sich den 4.-7. November 2020 frei und melden sich gleich auf https://www.dgnkongress.org/anmeldung an. Die DGN bietet Ihnen dieses technisch hochwertige, virtuelle Kongresserlebnis zu fairen Teilnahmegebühren. Diese beinhalten:

- alle virtuellen Veranstaltungsteile (Live-Veranstaltungen, Webcasts, E-Poster)

- Zugriff auf Webcasts, E-Poster und E-Learning für ein Jahr

\section{Fortbildung Live}

An allen vier Tagen gibt es analog $\mathrm{zu}$ „Wissenschaft Live“ ein interaktives Fortbildungsprogramm. Über diesen parallelen Live-Kanal werden ausgewählte Kurse der DGN-Fortbildungsakademie angeboten. Die Sessions bestehen in der Regel aus einem einstündigen Vortragsteil und einer anschließenden halbstündigen Q\&A-Runde.

Die Teilnehmer haben die Möglichkeit, ihre Fragen und Diskussionsbeiträge einzubringen, was eine interaktive Fortbildung ermöglicht. Das Themenspektrum ist breit gefächert und deckt die ganze Bandbreite der Neurologie ab. Das Programm finden Sie auf https://www.dgnkongress.org

\section{Junge Neurologen Live}

Am Freitag, den 6. November 2020, bespielen die Jungen Neurologen (JuNos) einen Live-Kanal und bieten Fortbildung von jungen Neurologinnen und Neurologen für junge Neurologinnen und Neurologen bzw. diejenigen, die es werden möchten. Das Programm finden Sie auf https:// www.dgnkongress.org

\section{Industrie Live}

Auf einem dritten Live-Kanal finden parallel zu den Kanälen Wissenschaft und Fortbildung Live die Industriesymposien statt. Das Programm wird inhaltlich von den Industriepartnern gestaltet. Das Programm finden Sie auf https://www.dgnkongress.org

Alle Kongressinhalte sind bis November 2021 abrufbar.
DGN-Mitglied Nichtmitglied

\begin{tabular}{|c|c|c|}
\hline Arzt/Ärztin & $90,00 €$ & $150,00 €$ \\
\hline Assistenzarzt/-ärztin & $75,00 €$ & $120,00 €$ \\
\hline ermäßigt* & $50,00 €$ & $80,00 €$ \\
\hline
\end{tabular}




\section{Kongress-Highlights im Überblick}

Der DGN-Kongress bietet zahlreiche Highlight-Veranstaltungen, die bereits bei den Präsenzkongressen in den Vorjahren überzeugt haben. Sie werden auch den ersten digitalen Kongress der DGN bereichern!

\section{Eröffnungsveranstaltung}

\section{November 2020,}

14.30-16.00 Uhr

Das beherrschende Thema der Zeit ist derzeit die SARS-CoV2-Pandemie. Sie hat die DGN sowie die gesamte Neurologie vor große Herausforderungen gestellt: Der Kongress musste auf ein digitales Format umgestellt werden, aber auch inhaltlich fand sich das Fach schnell im Zentrum der Pandemie, nachdem deutlich wurde, dass viele COVID-19-Patientinnen/Patienten neurologi-

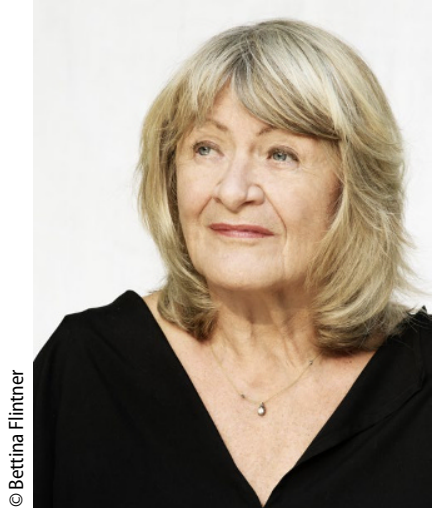

$\Delta$ Alice Schwarzer

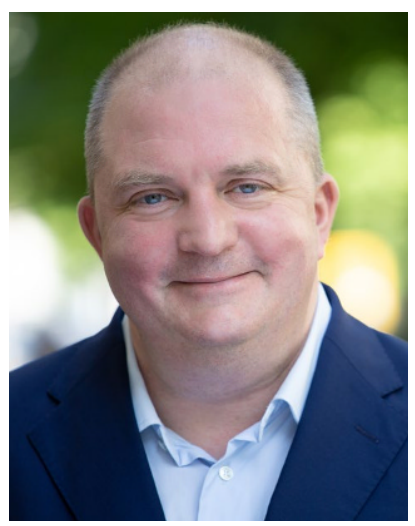

$\Delta$ Jörg Thadeusz moderiert die Eröffnungsveranstaltung

20.30-21.30 Uhr nungsveranstaltung wird DGNPräsidentin Prof. Christine Klein, Lübeck, über die Arbeit der DGN während der Corona-Pandemie berichten. Es wurde viel geleistet: Die DGN-Kommissionen haben Stellungnahmen und Empfehlungen $\mathrm{zu}$ ihren Gebieten erar- beitet und sie kontinuierlich aktualisiert, eine S1-Leitlinie zu neurologischen Manifestationen bei COVID-19-Patientinnen/Patienten wurde unter Federführung von DGN-Generalsekretär Professor Peter Berlit erarbeitet und publiziert, die Gremienarbeit wurde

\section{Mittwoch, 4. November 2020}

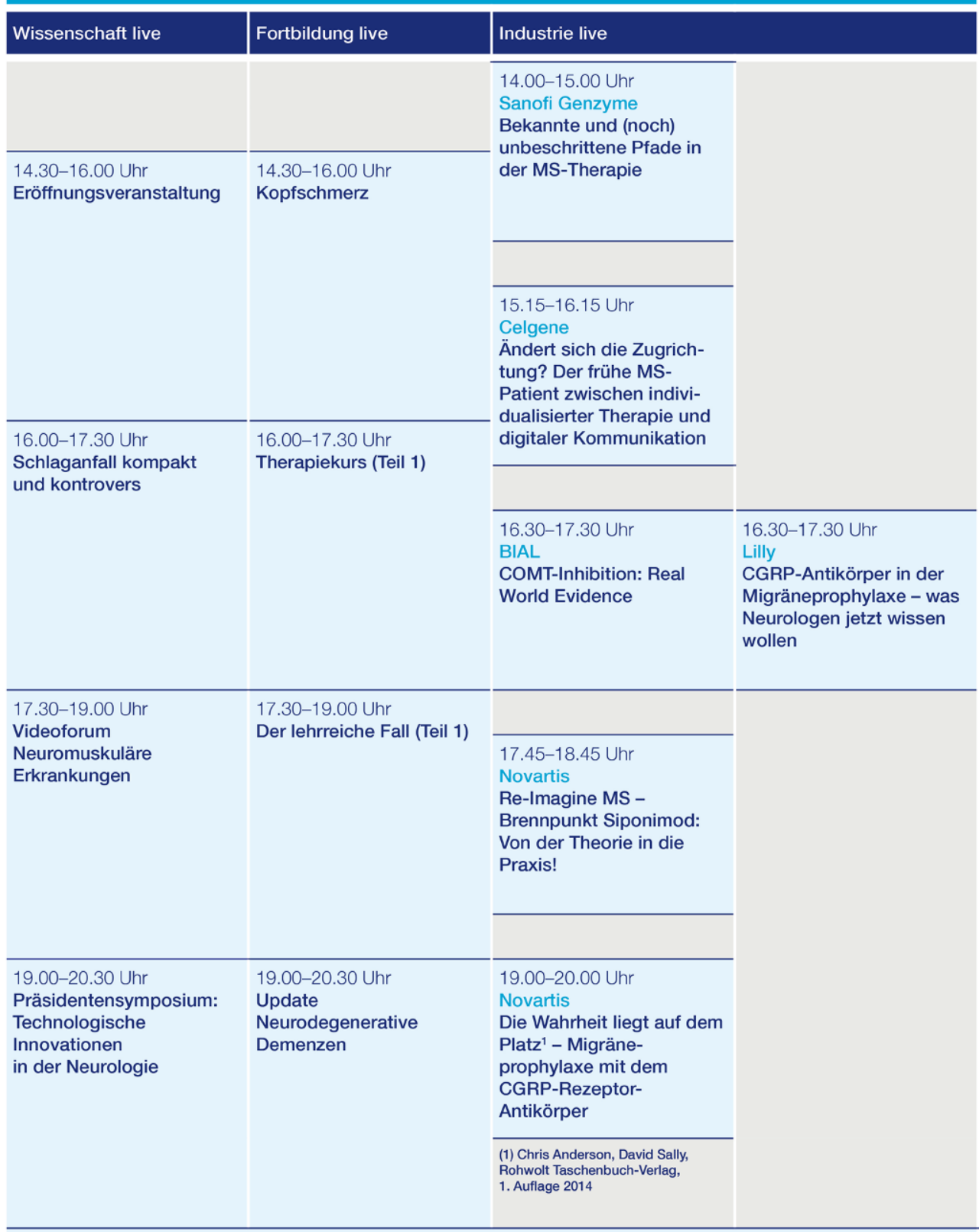

Remains of the Day - Abendliche Talkrunde 


\section{Donnerstag, 5. November 2020}

\begin{tabular}{|c|c|c|c|}
\hline Wissenschaft live & Fortbildung live & \multicolumn{2}{|l|}{ Industrie live } \\
\hline & & 15.45-16.45 Uhr & 15.45-16.45 Uhr \\
\hline \multirow[t]{2}{*}{$\begin{array}{l}\text { 16.00-17.30 Uhr } \\
\text { COVID-19 } \\
\text { in der Neurologie }\end{array}$} & \multirow[t]{2}{*}{$\begin{array}{l}\text { 16.00-17.30 Uhr } \\
\text { Therapiekurs (Teil 2) }\end{array}$} & $\begin{array}{l}\text { Spurensuche - auf der } \\
\text { richtigen Fährte! Von } \\
\text { häufigen Symptomen zu } \\
\text { seltenen Diagnosen }\end{array}$ & $\begin{array}{l}\text { Aktuelle Strategien zum } \\
\text { Management intra- } \\
\text { kranieller Blutungen } \\
\text { unter FXa-Inhibition }\end{array}$ \\
\hline & & $\begin{array}{l}\text { 17.00-18.00 Uhr } \\
\text { Bristol-Myers Squibb } \\
\text { und Pfizer }\end{array}$ & \\
\hline \multirow{2}{*}{$\begin{array}{l}17.30-19.00 \text { Uhr } \\
\text { Videoforum } \\
\text { Schwindel, Okulo- } \\
\text { motorikstörungen und } \\
\text { Nystagmus - „seeing is } \\
\text { believing“ }\end{array}$} & \multirow[t]{2}{*}{$\begin{array}{l}\text { 17.30-19.00 Uhr } \\
\text { Der lehrreiche Fall (Teil 2) }\end{array}$} & $\begin{array}{l}\text { Neue Wege im } \\
\text { Schlaganfall-Management }\end{array}$ & \\
\hline & & $\begin{array}{l}18.15-19.15 \text { Uhr } \\
\text { Novartis } \\
\text { Re-Imagine MS - } \\
\text { Brennpunkt Ofatumumab }\end{array}$ & \\
\hline \multirow{2}{*}{$\begin{array}{l}19.00-20.30 \text { Uhr } \\
\text { Highlights der klinischen } \\
\text { Neurologie: Neurology in } \\
\text { Progress }\end{array}$} & \multirow{2}{*}{$\begin{array}{l}\text { 19.00-20.30 Uhr } \\
\text { Epilepsie }\end{array}$} & & \\
\hline & & $\begin{array}{l}\text { 19.30-20.30 Uhr } \\
\text { Merck } \\
\text { Gerade jetzt: Neuro- } \\
\text { degeneration aufhalten - } \\
\text { früh die richtigen Impulse } \\
\text { setzen }\end{array}$ & \\
\hline
\end{tabular}

20.30-21.30 Uhr

Remains of the Day - Abendliche Talkrunde

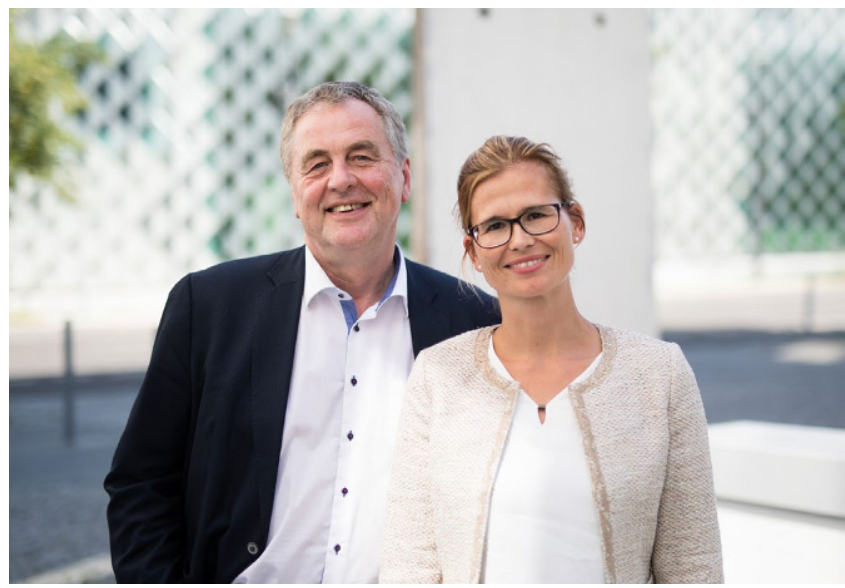

$\Delta$ Prof. Reetz und Prof. Erbguth

ad hoc auf „digital“ umgestellt und der Journal-Club-Newsletter, den Prof. Berlit wöchentlich herausgegeben hat, bot allen Mitgliedern einen schnellen Überblick über die aktuelle und rasant wachsende Studienlage zum Thema. Doch das wurde, um zu erheben, wie sich die Veränderungen in der aktuellen Situation auf die Stimmungslage der Neurologinnen und Neurologen ausgewirkt haben - über erste Ergebnisse wird Professor Christine Klein berichten.

Doch Corona ist nicht das einzige Thema: Das vergangene Jahr war wie kaum ein anderes durch die gesellschaftliche Diskussion um Geschlechtergerechtigkeit und Chancengleichheit von Frauen geprägt - und gleichzeitig war es das erste Jahr, in dem eine Frau an der Spitze der DGN stand. Das zeigt, dass die Neurologie ein modernes, zeitgemäßes Fach ist, zumal neben DGN-Präsidentin Prof. Christine Klein viele Neurologinnen hochrangige Positionen in Klinik, Praxis und Universität bekleiden. $\mathrm{Ob}$ das reicht und die Neurologie als Blaupause für andere Disziplinen herange- zogen werden kann, wird auf der Eröffnungsveranstaltung niemand Geringeres als die Frauenrechtlerin Alice Schwarzer beleuchten. Die streitbare Journalistin, Verlegerin und Publizistin wird einen Einblick in ihr spannendes Leben gewähren und einen Ausblick auf die Frauenbewegung im 21. Jahrhundert geben, dabei auch Analogien zur Neurologie aufmachen. Was wurde schon erreicht? Und was muss noch getan werden? Wie sind der Stand der neurologischen Gender-Forschung und die Rolle der Frauen in der Neurologie? Das sind Diskussionspunkte, die Alice Schwarzer zusammen mit Prof. Christine Klein in einem Talkrunden-Format diskutiert wird. Moderiert wird der Talk von Dr. Wolfgang Niess, Historiker und bis 2018 leitender Redakteur beim SWR Fernsehen.

Und noch ein drittes Themenhighlight erwartet Sie bei der Eröffnungsveranstaltung: Mit dem Kongress 2020 fällt auch der Startschuss für die Deutsche Hirnstiftung, die neurologische Themen in die breite Öffentlichkeit tragen wird. Ihre Aufgabe wird es sein, die Bevölkerung umfassend, objektiv und frei von Interessenkonflikten über neurologische Erkrankungen, ihre Therapien und vor allem auch Möglichkeiten der Prävention $\mathrm{zu}$ informieren. Dazu wird eine Gesprächsrunde mit Professor Kathrin Reetz und Professor Frank Erbguth stattfinden, die das ambitionierte Projekt für die deutsche Neurologie umsetzen. Durch die Eröffnungsveranstaltung wird der bekannte Moderator Jörg Thadeusz führen - schnell, schlagfertig und dabei doch immer charmant.

\section{COVID-19 in der Neuro- logie}

\section{November 2020, 16.00-17.30 Uhr}

Die SARS-CoV-2-Pandemie hat die DGN und die gesamte Neurologie vor große Herausforderungen gestellt: Der Kongress musste auf ein digitales Format umgestellt werden, aber auch 


\begin{tabular}{|c|c|c|c|c|}
\hline \multirow[t]{2}{*}{ Wissenschaft live } & \multirow[t]{2}{*}{ Fortbildung live } & \multicolumn{2}{|l|}{ Industrie live } & \multirow[t]{2}{*}{ Special: JuNos live } \\
\hline & & \multirow{2}{*}{$\begin{array}{l}15.45-16.45 \text { Uhr } \\
\text { Teva } \\
\text { Behandlung der MS } \\
\text { im Lebensbogen }\end{array}$} & & \\
\hline \multirow[t]{2}{*}{$\begin{array}{l}16.00-17.30 \text { Uhr } \\
\text { Videoforum } \\
\text { Epileptische Anfälle und } \\
\text { Differenzialdiagnosen }\end{array}$} & \multirow[t]{2}{*}{$\begin{array}{l}16.00-17.30 \text { Uhr } \\
\text { Therapiekurs (Teil 3) }\end{array}$} & & & \multirow[t]{2}{*}{$\begin{array}{l}\text { 16.00-17.30 Uhr } \\
\text { Meet the Expert: } \\
\text { Neuroprotektive und } \\
\text { regenerative } \\
\text { Therapiestrategie }\end{array}$} \\
\hline & & \multirow[t]{2}{*}{$\begin{array}{l}17.00-18.00 \text { Uhr } \\
\text { Teva } \\
\text { CGRP-Science-Slam: } \\
\text { aus der Wissenschaft } \\
\text { in die Klinik }\end{array}$} & \multirow[t]{2}{*}{$\begin{array}{l}17.00-18.00 \mathrm{Uhr} \\
\text { Alexion } \\
\text { Neuroimmunologie - } \\
\text { Innovation und } \\
\text { Wirtschaftlichkeit }\end{array}$} & \\
\hline \multirow[t]{2}{*}{$\begin{array}{l}\text { 17.30-19.00 Uhr } \\
\text { Neurologie und Neurologen } \\
\text { im Nationalsozialismus }\end{array}$} & \multirow[t]{2}{*}{$\begin{array}{l}\text { 17.30-19.00 Uhr } \\
\text { Bewegungsstörungen }\end{array}$} & & & \multirow[t]{2}{*}{$\begin{array}{l}17.30-19.00 \text { Uhr } \\
\text { Global Neurology }\end{array}$} \\
\hline & & \multirow[t]{2}{*}{$\begin{array}{l}18.15-19.15 \text { Uhr } \\
\text { Pfizer } \\
\text { Polyneuropathie - } \\
\text { Diagnostik mit } \\
\text { Konsequenzen }\end{array}$} & & \\
\hline \multirow[t]{2}{*}{$\begin{array}{l}19.00-20.30 \text { Uhr } \\
\text { Neuroimmunologie }\end{array}$} & \multirow[t]{2}{*}{$\begin{array}{l}\text { 19.00-20.30 Uhr } \\
\text { Schlaganfall }\end{array}$} & & & \multirow[t]{2}{*}{$\begin{array}{l}\text { 19.00-20.30 Uhr } \\
\text { Neurologischer Notfall }\end{array}$} \\
\hline & & $\begin{array}{l}\text { 19.30-20.30 Uhr } \\
\text { Arvelle } \\
\text { Pharmakoresistente fokale } \\
\text { Epilepsien: herausfordernd } \\
\text { für Ärzte, belastend und } \\
\text { risikoreich für Patienten - } \\
\text { ein Update zur Bedeutung } \\
\text { und zum therapeutischen } \\
\text { Management }\end{array}$ & & \\
\hline
\end{tabular}

20.30-21.30 Uhr

Remains of the Day - Abendliche Talkrunde

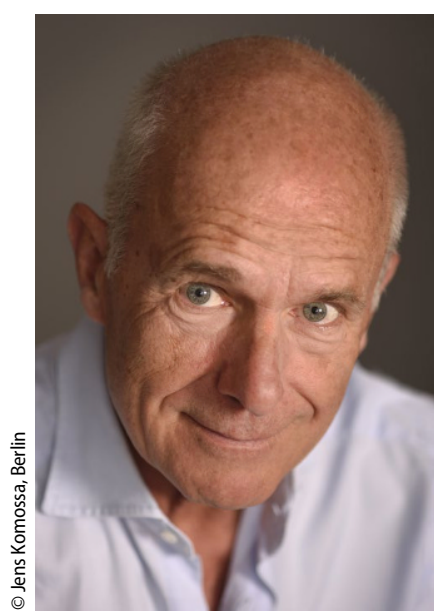

$\Delta$ Professor Peter Berlit

inhaltlich fand sich das Fach schnell im Zentrum der Pande- mie, nachdem deutlich wurde, dass viele COVID-19-Patientinnen/Patienten neurologische Symptome entwickeln, z. T. auch neurologische Spätfolgen.

DGN-Generalsekretär Professor Peter Berlit hat sich besonders intensiv mit den neurologischen Aspekten von COVID-19 auseinandergesetzt, er ist federführender Autor der S1-Leitlinie „Neurologische Manifestationen bei COVID-19-Patientinnen/ Patienten" und gibt darüber hinaus den DGN-JournalclubNewsletter zu SARS-CoV-2 heraus, der seit April wöchentlich erscheint.

Auf dem Kongress wird er eine Veranstaltung ausrichten, in

\section{Highlights der klinischen Neurologie: „Neurology in Progress"}

\section{November 2020, 19.00-20.30 Uhr}

Wissenschaftliche „Druckbetankung ", um up to date zu bleiben: DGN-Pressesprecher Prof. Dr. Hans-Christoph Diener stellt die wichtigsten klinischen Studien in der Neurologie aus dem Zeitraum September 2019 bis Anfang November 2020 vor und erklärt, wie sich die Ergebnisse der Studien in der klinischen Praxis auswirken. Die letzten $30 \mathrm{Minu}$ ten sind für Diskussion und Fragen vorgesehen.

\section{Neurologie und Neuro- logen im Nationalsozia- lismus mit anschließender Diskussionsrunde}

\section{November 2020, 19.00- 20.30 Uhr}

Im Supplement „Neurologen und Neurowissenschaftler in der NSZeit" (Beilage zu „Der Nervenarzt" 2/2020) wurde die Täterseite beleuchtet und die Vitae von belasteten Persönlichkeiten in der Neurologie aufgerollt. In diesem Symposium soll nun eine Auseinandersetzung mit den Opfern stattfinden. Vortragsthemen sind „Legalisierte Entrechtung: Rahmenbedingungen von Entlassung und Vertreibung im Nationalsozialismus" (Heiner Fangerau/Michael Martin) und „Sir Ludwig Guttmann: Ein vertriebener Neurologe als späteres Ehrenmitglied der DGN“ (Axel Karenberg/Michael Martin). Das Symposium unter dem Vorsitz von Prof. Dr. Martin Grond, Siegen, und Prof. Dr. Axel Karenberg, Köln, lädt zur Diskussion ein.

Im Anschluss sollen auch die Resultate des Moratoriums diskutiert werden, welches Anfang des Jahres beschlossen wurde, um eine sorgfältige historische Aufarbeitung aller Persönlichkeiten, nach denen Wissenschaftspreise und Ehrungen benannt wurden, zu leisten.

Wie aktuell das Thema auch heute noch ist, zeigt die lebhafte 


\section{Samstag, 7. November 2020}

\begin{tabular}{|c|c|c|c|}
\hline \multirow[t]{2}{*}{ Wissenschaft live } & \multirow{2}{*}{ Fortbildung live } & \multicolumn{2}{|l|}{ Industrie live } \\
\hline & & \multirow{2}{*}{$\begin{array}{l}09.15-10.15 \text { Uhr } \\
\text { Roche } \\
\text { Challenge accepted! - } \\
\text { Progression unterdrücken: } \\
\text { der Schlüssel in der } \\
\text { modernen MS-Therapie }\end{array}$} & \multirow{2}{*}{$\begin{array}{l}\text { 09.15-10.15 Uhr } \\
\text { Biogen } \\
\text { Kleine Sequenz - große } \\
\text { Wirkung: Nusinersen zur } \\
\text { Behandlung der } 5 \mathrm{q}-\mathrm{SMA} \text { als } \\
\text { Vorreiter für zukünftige ASO- } \\
\text { Innovationen bei neurodege- } \\
\text { nerativen Erkrankungen }\end{array}$} \\
\hline $\begin{array}{l}09.30-11.00 \text { Uhr } \\
\text { Pathophysiologie und } \\
\text { Klinik der Demenzen }\end{array}$ & \multirow[t]{2}{*}{$\begin{array}{l}09.30-11.00 \text { Uhr } \\
\text { Nerv und Muskel: } \\
\text { Neues zu erworbenen und } \\
\text { erblichen Myopathien } \\
\text { und Neuropathien }\end{array}$} & & \\
\hline & & \multirow{2}{*}{$\begin{array}{l}\text { 10.30-11.30 Uhr } \\
\text { Roche } \\
\text { Transforming } \\
\text { Neuroscience- } \\
\text { Erhalten, was uns } \\
\text { ausmacht }\end{array}$} & \\
\hline \multirow[t]{2}{*}{$\begin{array}{l}11.00-12.30 \text { Uhr } \\
\text { Videoforum } \\
\text { Bewegungsstörungen }\end{array}$} & \multirow[t]{2}{*}{$\begin{array}{l}11.00-12.30 \mathrm{Uhr} \\
\text { Multiple Sklerose }\end{array}$} & & \\
\hline & & $\begin{array}{l}\text { 11.45-12.45 Uhr } \\
\text { Alnylam } \\
\text { Multidisziplinär gelöst - } \\
\text { Hereditäre } \\
\text { Polyneuropathie }\end{array}$ & \\
\hline $\begin{array}{l}12.30-14.00 \mathrm{Uhr} \\
\text { Spotlight: } \\
\text { Höhepunkte des } \\
\text { Kongresses }\end{array}$ & $\begin{array}{l}12.30-14.00 \text { Uhr } \\
\text { Schwindel und Augen- } \\
\text { bewegungsstörungen - } \\
\text { Diagnose und Therapie }\end{array}$ & & \\
\hline
\end{tabular}

Diskussion innerhalb der Fachgesellschaft. Nach Publikation des Supplements erreichten uns viele Leserbriefe von DGN-Mitgliedern. Ein Teil der Briefe wurde bereits in der Ausgabe 6 von „Der Nervenarzt“ publiziert, weitere Leserstimmen finden Sie in dieser Ausgabe.

\section{Spotlight - Höhepunkte des Kongresses}

\section{November 2020,}

12.30-14.00 Uhr

Wie sagt man so schön - das Beste kommt zum Schluss. Unter dem Vorsitz von DGN-Präsidentin Prof. Dr. Christine Klein, Lübeck, und Kongresspräsident
Prof. Dr. Matthias Endres, Berlin, fassen Experten des jeweiligen Fachgebiets die Höhepunkte des wissenschaftlichen Programms zusammen. Folgende Themenschwerpunkte werden von den Experten beleuchtet: Schlaganfall, Epilepsie, Multiple Sklerose, Bewegungsstörungen sowie Altern und demenzielle Erkrankungen.

\section{Videoforen}

Das Veranstaltungsformat des Videoforums ist weit mehr als nur eine mediale, dem Zeitgeist geschuldete Spielerei. Es hat sich durchgesetzt, weil gerade in der Neurologie die phänomenologi- sche Befundung eine große Rolle spielt und Videokasuistiken einen hohen Lehrgehalt haben. Videoforen sind damit innovative Formate der Weiterbildung, die sich darüber hinaus in einem besonderen Maße für einen Online-Kongress anbieten.

Bitte beachten Sie: Die Videoforen stehen exklusiv nur den Live-Zuschauern zur Verfügung.

\section{Neuromuskuläre Erkran- kungen}

\section{November 2020,} 17.30-19.00 Uhr

Das Videoforum soll interaktiv eine Auswahl von Fäl- len genetisch bedingter sowie erworbener, nicht erblicher neuromuskulärer Erkrankungen wie Polyneuropathien, Motoneuronerkrankungen, Myopathien, Muskeldystrophien und lysosomale Speichererkrankungen vorstellen. Dabei liegt der Schwerpunkt auf charakteristischen Anamneseberichten, typischen klinischen Untersuchungsbefunden und deren Differenzialdiagnosen.

Die Vorsitzenden dieses Videoforums sind Jun.-Prof. Dr. Elena Enax-Krumova, Bochum, und Prof. Dr. Matthias Vorgerd, Bochum.

\section{Schwindel, Okulomo- torikstörungen und Nystagmus - „seeing is believing"}

\section{November 2020, 17.30-19.00 Uhr}

Bei Augenbewegungsstörungen, Nystagmus und Schwindelsyndromen ist oft buchstäblich eine rasche Blickdiagnose möglich. Deshalb eignet sich dieses Thema auch hervorragend für ein virtuelles Videoforum, um den diagnostischen Blick alias Mustererkennung der Teilnehmer weiter zu schärfen. Die richtige Einordnung dieser Störungen erlaubt oft eine genaue topographische Diagnose: Handelt es sich um eine periphere oder zentrale Läsion im Bereich von Hirnstamm oder Kleinhirn? Interaktiv werden in 90 Minuten die klinisch relevantesten Befunde dargestellt.

Die Vorsitzenden dieses Videoforums sind Prof. Dr. Michael Strupp, München, Prof. Dr. Dominik Straumann, Zürich/ Schweiz, und PD Dr. Andreas Zwergal, München.

\section{Epileptische Anfälle und Differenzialdiagnosen}

\section{November 2020, 16.00-17.30 Uhr}

Es werden Beiträge zu unterschiedlichen Anfallstypen bei Frontallappenepilepsien vorgestellt. Basierend auf der Anfalls- 


\section{Kongress-TV: Neurologie strahlt aus - weit über den Fachkreis!}

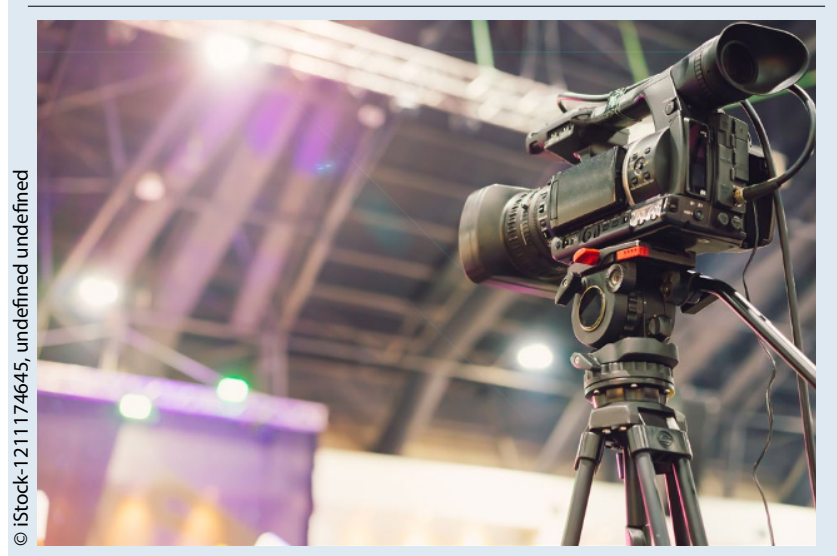

Mit dem Kongress-TV möchten wir neurologische Inhalte über die Fächergrenzen hinaus transportieren: Wir sprechen damit Medizinerinnen und Mediziner anderer Fächer an, Pflegekräfte, Patientinnen/ Patienten, Angehörige, aber auch Medienmacher und Gesundheitspolitiker. Die Neurologie bietet spannende Themen für die Öffentlichkeit - und wir möchten diese in verschiedenen Formaten aufbereiten: kleine Features, Experteninterviews, Talkrunden - oder der Blick „backstage" bei einem virtuellen Kongress. Dieser Content ist für alle ohne Login zugänglich. 2020, aber auch bei den kommenden Präsenzkongressen wird das Angebot um Expertengespräche und kurze Reportagen ergänzt. Federführend wird dieser Kanal durch die Pressestelle der DGN bestückt. semiologie, werden der Ort des Anfallsursprungs und ggf. Wege der Anfallspropagation aufgezeigt und diskutiert. Die exemplarischen Anfallsvideos werden im Kontext des jeweiligen Krankheitsbildes präsentiert. Ziel des Videoforums ist das Erkennen von epileptischen Anfällen aus dem Frontallappen und das Verständnis der der Semiologie zugrunde liegenden funktionellen Neuroanatomie.

Die Vorsitzenden dieses Videoforums sind Prof. Dr. Martin Holtkamp, Berlin, und Prof. Dr. Christian G. Bien, Bielefeld.

\section{Videoforum Bewegungs- störungen}

\section{November 2020, \\ 11.00-12.30 Uhr}

Das Format eines Videoforums bietet sich gerade für das Erlernen/Vertiefen von Diagnosen im Bereich von Bewegungsstörungen an. Eingangs hält Prof. Dr.
Präsident Prof. Christian Gerloff, Past-Präsident Prof. Gereon Fink, Prof. Martin Grond, Leiter der DGN-Fortbildungsakademie, und Kongresspräsident Prof. Matthias Endres) zusammen mit einem Gastexperten den Tag auswerten, die Essenz für Klinik und Praxis herausarbeiten und kontrovers diskutieren. Ob Sie die Veranstaltung im "Arbeitsmodus“ im Büro oder als Feierabendevent mit einem Glas Wein auf der Couch verfolgen, bleibt Ihnen überlassen - die Grundbedürfnisse beider Rezeptionsformen, Informationsgewinn und Entertainment, kommen in keinem Fall zu kurz!

\section{BDN-Forum 2020 im Rahmen des DGN-Kongresses}

\section{Strategiewechsel jetzt! Brauchen wir neue Allianzen unter stärkerer Beteiligung der deutschen Ärzteschaft für eine gute Medizin?}

Die Coronakrise hat einmal mehr deutlich gemacht, dass das Verhältnis von Ökonomie und Medizin neu überdacht werden muss. Dr. Klaus Reinhardt, Präsident der Bundesärztekammer, sagt hierzu: „Kliniken sind Einrichtungen der Daseinsfürsorge und keine Industriebetriebe, die sich ausschließlich an Rentabilitätszahlen ausrichten“.

Bereits 2013 haben die Delegierten auf dem 116. Deutschen Ärztetag mit großer Mehrheit dem Leitantrag „Menschen statt Margen in der Medizin“ zugestimmt. Demnach forderte die Ärzteschaft, das Gesundheitssystem stärker an den Bedürfnissen der Patienten, statt an rein öko- nomischen Prinzipien auszurichten. Die Sorge galt auch der steigenden Zahl der Behandlungsfälle in Klinik und Praxis bei gleichzeitiger Rückläufigkeit der zur die Verfügung stehenden Arztstunden, der Zunahme des ökonomischen Drucks durch fortwährende Unterfinanzierung, die mit Verdichtung von Arbeit und Überlastung einhergeht. Das ist sieben Jahre her. Inzwischen hat sich das Bündnis „Junge Ärzte“" gegründet, welches zusammen mit dreiundzwanzig unterstützenden Verbänden ein Ende der Profitmaximierung fordert, und sich für eine „Medizin für den Menschen“ und für „Gesundheit vor Gewinn“ stark macht.

2019 hat eine größere Gruppe von Ärzten in Zusammenarbeit mit zahlreichen Fachgesellschaften die Forderungen an die Öffentlichkeit gerichtet, Gesundheitsversorgung nach dem

\section{Remains of the Day - Abendliche Talkrunde}

\section{4., 5. und 6. November, 20.30-21.30 Uhr}

Was vom Tage übrig bleibt: allabendliche Auswertung der in den Live-Sessions vorgestellten Daten und erfolgten Diskussionen - fundiert und gleichzeitig höchst unterhaltsam. Genießen Sie dieses Edutainment-Format, bei dem fünf Experten (DGNPräsidentin Prof. Christine Klein, Stellvertretender DGN-
Datum: 5. November, 14.30-15.30 Uhr

Ort*: Hörsaal der Kaiserin Friedrich-Stiftung, Robert-Koch Platz 7, 10115 Berlin

*Da aufgrund der aktuellen Hygienemaßnahmen nur eine begrenzte Anzahl von Personen an der Veranstaltung teilnehmen können, wird das BDN-Forum in diesem Jahr als Hybrid-Veranstaltung angeboten. Das BDN-Forum wird live über das Kongressportal übertragen.

Referent: Dr. Klaus Reinhardt, Präsident der Bundesärztekammer

Diskussion: Dr. med. Uwe Meier, 1. Vorsitzender BDN, Präsident Spitzenverband ZNS

Prof. Dr. Martin Südmeyer, 2. Vorsitzender BDN

Moderation: Dr. Dr. Klaus Piwernetz, medimaxx health management $\mathrm{GmbH}$ 
Bedarf der Patienten und nicht nach betriebswirtschaftlichen Anreizen auszurichten. „Rettet die Medizin“ lautete der Appell und intendierte insbesondere die Inaugenscheinnahme des Fallpauschalensystems zur Krankenhausfinanzierung.

Umgekehrt bietet die Ökonomie die Grundlage für eine anspruchs- und qualitätsvolle medizinische Versorgung. Hier sind auch die Ärzte in der Pflicht, verantwortlich zu handeln und Ressourcen sinnvoll und gezielt einzusetzen. Bei der Frage der Gewichtung, etwa was möchte sich die Gesellschaft generell an medizinischem Standard leisten, braucht es hingegen auch einen Konsens, den die Politik verantworten muss. Den hierfür notwendigen gesellschaftlichen Diskurs scheuen Politiker und Ärzte aber oftmals. So sehen wir gerade in der Neurologie rasante medizinische Fortschritte, die die Gesellschaft aber auch vor ungeahnte ökonomische Herausforderungen stellt. $\mathrm{Zu}$ denken ist hier beispielsweise an zukünftige Antikörpertherapien von Demenzen.

Nach Corona ist vieles nicht mehr so wie vorher. Sollten wir dies nicht zum Anlass nehmen, uns als Ärzte, d.h. als Experten für Gesundheit, mehr in die politischen Diskussionen einzubringen? Braucht es neue Allianzen zwischen Ärzten und verantwortungsvollen Ökonomen jenseits von Partialinteressen?

Wir freuen uns außerordentlich, diese und andere Fragen zu beleuchten, indem wir den Präsidenten der Bundesärztekammer, Dr. Klaus Reinhardt, gewinnen konnten. Ferner wird Klaus Piwernetz aus seiner aktuellen Buchpublikation „Strategiewechsel jetzt!" berichten, so dass es zu einer spannenden Diskussion und einem regen Gedankenaustausch kommen wird.

Hierzu laden wir Sie herzlich ein!

\section{Dr. Uwe Meier und}

Prof. Dr. Martin Südmeyer mittels Neuromodulation künftig verschiedene Hirnerkrankungen behandeln lassen könnten. Der Pionier der Teleneurologie, Professor Lee H. Schwamm, Professor für Neurologie an der Harvard Medical School, skizziert, wie telemedizinische Verfahren die Gesundheitsversorgung eines Landes revolutionieren und gleichzeitig Kosten sparen.

\section{„Neuromodulation ist ein vielversprechendes Werk- zeug zur individualisierten Therapie von Bewegungs- störungen"}

Das Potenzial der tiefen Hirnstimulation ist revolutionär. Sogenannte Hirnschrittmacher verbessern schon heute die Lebensqualität von Menschen mit Parkinson und Dystonie. Andrea Kühn will das grundlegende Prinzip der Neuromodulation künftig auch auf andere Hirnerkrankungen wie Schlaganfall und Tremor übertragen.

Frau Professor Kühn, was treibt Sie als Neurologin an?
Die Aussicht, dass wir durch ein tieferes Verständnis der pathophysiologischen Ursachen Patientinnen/Patienten mit verschiedenen Hirnerkrankungen in nicht allzu ferner Zukunft mittels Neuromodulation, also der gezielten Stimulation von Nervenzellen, eine individualisierte Therapie anbieten können, die ihre Lebensqualität maßgeblich verbessert.

Warum sollte man mittels Neuromodulation so unterschiedliche Bewegungsstörungen wie bei Parkinson, Tourette oder Trauma behandeln können?

Wir gehen heute davon aus, dass Bewegungsstörungen bei neurologischen Erkrankungen Ausdruck einer Netzwerkstörung sind - also einer gestörten Kommunikation zwischen verschiedenen motorischen Hirnregionen. Mittels invasiver und nicht invasiver elektrischer oder magnetischer Stimulation lässt sich die Aktivität des neuronalen Netzwerks gezielt beeinflussen: Pathologische Netzwerkaktivitäten werden unterdrückt und phy-

\section{Präsidentensymposium: Science-Fiction wird klinische Realität}

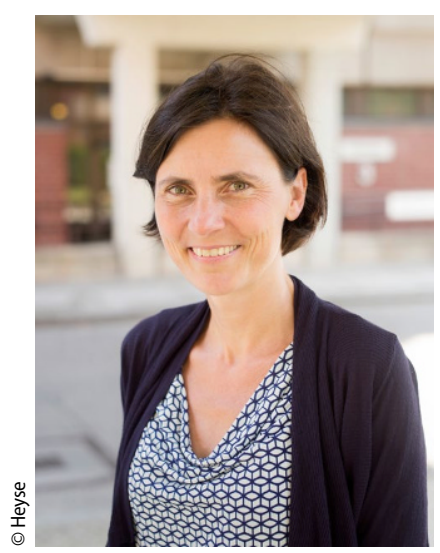

$\Delta$ Prof. Dr. Andrea Kühn, Leiterin der Sektion Bewegungsstörungen und Neuromodulation an der Charité, berichtet auf dem Präsidentensymposium über bedarfsgerechte Closed-Loop-Stimulation
"Auf dem Präsidentensymposium am 4. November 2020 berichten international renommierte Experten ab 19 Uhr über neueste Entwicklungen in ihrem Fachgebiet, die für Außenstehende noch wie Science-Fiction anmuten“, freut sich Kongresspräsident Professor Matthias Endres. Professor Grégoire Courtine und Professor Jocelyne Bloch arbeiten daran, die Lebensqualität von Patientinnen und Patienten mit schweren Rückenmarksverletzungen zu verbessern (wir berichteten). Professor Andrea Kühn, Leiterin der Sektion Bewegungsstörungen und Neuromodulation an der Klinik für Neurologie der Charité, zeigt, wie sich

\section{Über Professor Andrea Kühn}

Seit 2016 leitet Prof. Dr. Andrea Kühn die Sektion Bewegungsstörungen und Neuromodulation am Campus Charité Mitte und Campus Virchow-Klinikum. Die Neurologin ist außerdem Sprecherin des neu bewilligten überregionalen Sonderforschungsbereichs (SFB/Transregio) „Behandlung motorischer Netzwerkstörungen mittels Neuromodulation", der von der Charité und der Universitätsklinik Würzburg getragen wird.

Prof. Kühns Schwerpunkt liegt sowohl klinisch als auch wissenschaftlich in der Behandlung von Bewegungsstörungen mit dem Verfahren der tiefen Hirnstimulation. Ihr Ziel ist es, die Mechanismen der tiefen Hirnstimulation zu entschlüsseln und zu nutzen, um die Therapie zu optimieren. Ihr Team befasst sich vor allem mit der Charakterisierung von Physiomarkern in lokalen Feldpotenzialen bei Patientinnen/Patienten mit Bewegungsstörungen wie dem idiopathischen ParkinsonSyndrom. Den Grundstein für die Forschung stellt die pathologisch erhöhte Synchronisierung im Beta-Frequenzbereich der Basalganglien-Kortex-Schleife dar. Dieser Physiomarker korreliert mit der Schwere von Parkinson-Symptomen wie verlangsamte Bewegungen und Steifigkeit und gilt mittlerweile als etabliert. Prof. Kühns Arbeiten in Zusammenarbeit mit Peter Brown, University of Oxford, gelten hierfür als wegbereitend. Darauf aufbauend, arbeitet das Team um Andrea Kühn an der Entwicklung von sogenannten Closed-Loop-Systemen im Bereich der tiefen Hirnstimulation. Zusätzlich beschäftigen sich die Forscher methodisch mit Elektroenzephalographie, funktioneller Magnetresonanztomographie, diffusionsgewichteter Fasertraktographie und struktureller Magnetresonanztomographie. 

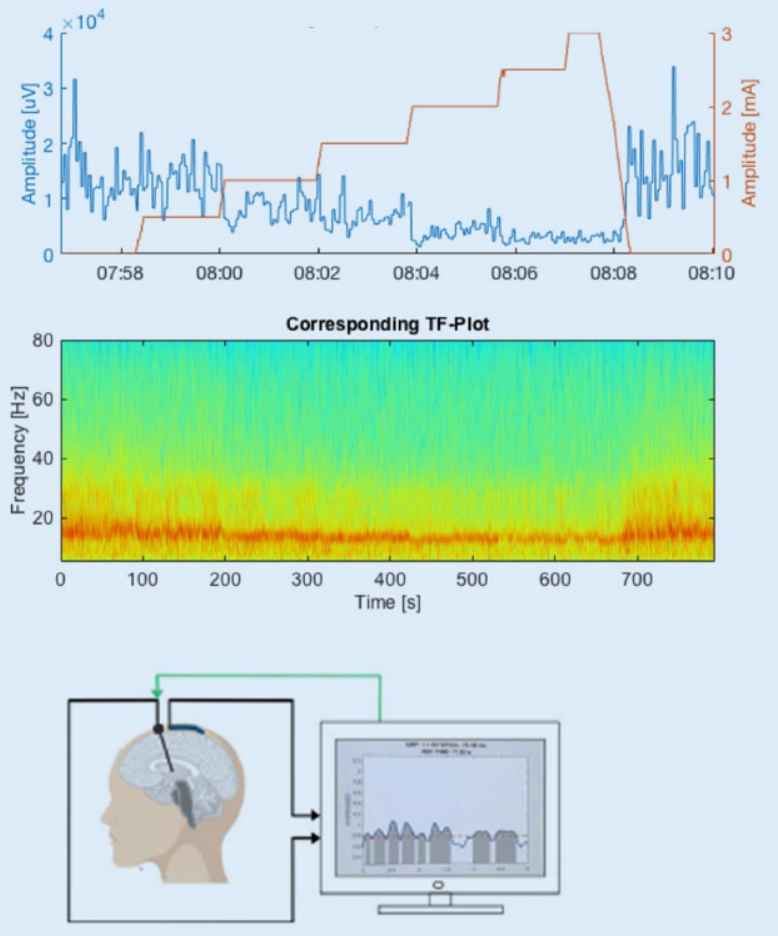

\ Stimulationsbedingte Suppression pathologischer Aktivität im Ncl. subthalamicus bei einem Patienten mit M. Parkinson. Die obere Abbildung zeigt die gefilterte Beta-Band-Aktivität (blau) unter steigender Stimulationsintensität (rot), die mittlere die Power im gesamten Spektrum über die Zeit der Stimulation. Unten: adaptive Stimulation mit Modulation der Stimulationsamplitude durch die Anpassung an die Beta-Band-Aktivität

siologische verstärkt. So lässt sich die gestörte Hirnfunktion wiederherstellen.

Bei welchen neurologischen Erkrankungen wird Neuromodulation bereits erfolgreich eingesetzt?

Patientinnen/Patienten mit Parkinson-Syndrom, Dystonie und Tremor therapieren wir bereits erfolgreich mittels tiefer Hirnstimulation (THS), in Studien gab es gute Erfolge bei Patientinnen/Patienten mit Huntington und Tourette. Hier können wir die motorischen Symptome wie die unwillkürlichen Bewegungen lindern, die sich durch Stimulation unterdrücken lassen. Die Grenzen: Bei einigen Behandelten bleibt der Erfolg der THS hinter den Erwartungen zurück, und auf viele andere Hirnerkrankungen, zum Beispiel psychiatrische Krankheiten, Schlaganfall oder Trauma, lässt sich die Therapie bislang nicht übertragen.
Warum sind die Prinzipien der Neuromodulation bislang nur auf wenige Hirnerkrankungen übertragbar?

Wir haben den Mechanismus und die Grundlagen anderer Hirnerkrankungen noch nicht weit genug verstanden, um die THS auf sie übertragen zu können. Wir müssen unser Verständnis von Netzwerkstörungen erst einmal noch vertiefen, um auch auf der zellulären Ebene zu erfassen, wie der Krankheitsmechanismus letztlich $\mathrm{zu}$ einer Veränderung des Netzwerks führt. Untersuchen müssen wir auch die Mechanismen, die der Neuromodulation bei verschiedenen Erkrankungen zugrunde liegen. Warum reagieren Patientinnen/Patienten individuell unterschiedlich auf die THS? Wo im Gehirn liegt, je nach Erkrankung, der ideale Zielort für die Stimulation? Diese Fragen soll ein neues überregionales Verbundprojekts beantworten: der

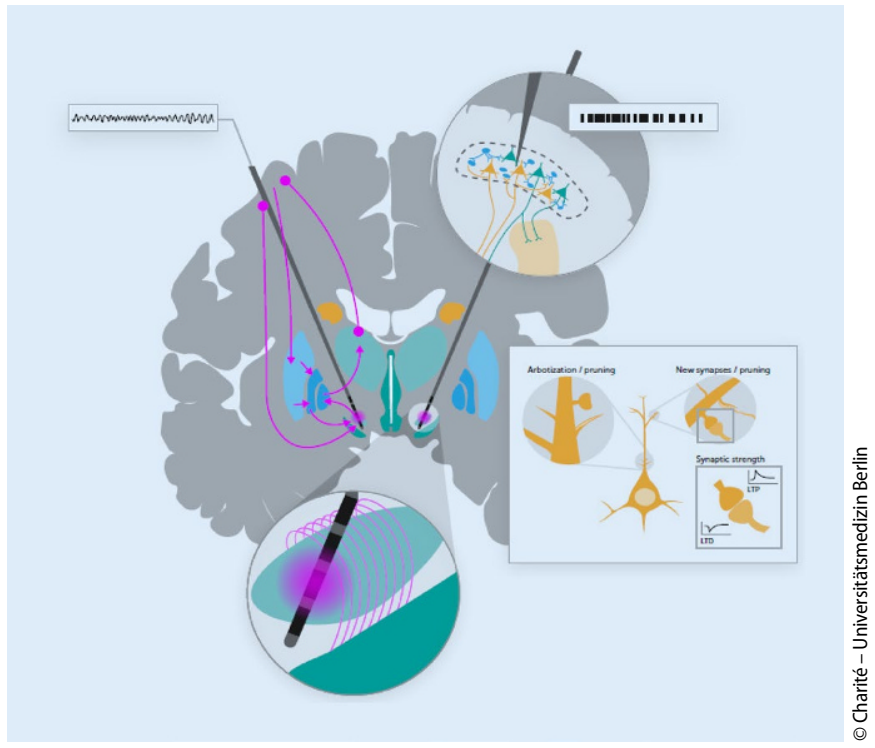

$\Delta$ Innovative Neuromodulationsstrategien: Der SFB/Transregio „Behandlung motorischer Netzwerkstörungen mittels Neuromodulation“ untersucht die vielfältigen Symptome von Erkrankungen motorischer Regelkreise durch Charakterisierung symptomspezifischer Netzwerkaktivität. Diese Erkenntnisse sollen genutzt werden, um innovative Neuromodulationsstrategien zu entwickeln. Prof. Kühn: „Unsere Vision sind klinische Neuromodulationsverfahren, die gezielt krankhaft veränderte Netzwerkaktivität ausschalten, aber normale Funktionen erhalten oder sogar wiederherstellen." Dabei fokussiert der SFB auf neurobiologische Mechanismen der tiefen Hirnstimulation auf zellulärer Ebene (Area A), Interaktionen in symptomspezifischen neuronalen Netzwerken (Area B) und erste klinische Anwendungen (Area C)

Sonderforschungsbereich (SFB/ Transregio) „Behandlung motorischer Netzwerkstörungen mittels Neuromodulation", der von der Charité und der Universitätsklinik Würzburg getragen und von der Deutschen Forschungsgemeinschaft mit zehn Millionen Euro gefördert wird.

Sie sind seit Kurzem Sprecherin des neuen SFB/Transregio - welche Schwerpunkte setzt das Forschungsprojekt?

Unser Ziel ist es, mögliche Angriffspunkte für eine Neuromodulation bei verschiedenen neurologischen Erkrankungen $\mathrm{zu}$ finden und krankheitsbedingte Veränderungen näher $\mathrm{zu}$ bestimmen. Unsere Vision: möglichst rasch Neuromodulationsverfahren für die klinische Praxis zu entwickeln, die netzwerkspezifisch ansetzen, um komplexe klinische Syndrome behandeln zu können. So ein Forschungsprojekt lässt sich nur umsetzen, wenn, wie in unserem
SFB/Transregio, international ausgewiesene Grundlagenwissenschaftler, Experten für digitale Medizin und Kliniker zusammenarbeiten - das macht diese Verbundinitiative weltweit einmalig.

Die grundlegende Idee ist, bedarfsgerecht pathologische Netzwerkaktivitäten $\mathrm{zu}$ unterdrücken und physiologische zu verstärken, um so Krankheitssymptome zu mildern und Nebenwirkungen zu vermeiden. Ein Schwerpunkt unserer Forschung ist deshalb die Entwicklung bedarfsgesteuerter Schrittmachersysteme, sogenannter Closed-Loop-Systeme, die nur bei Auftreten von Krankheitssymptomen aktiv werden. Wo wir damit aktuell stehen und welche Möglichkeiten für die Therapie mittels tiefer Hirnstimulation sich künftig eröffnen, werde ich auf dem Präsidentensymposium berichten. Ein zweiter Aspekt sind MRT-gestützte Darstellungen der neuronalen Netzwerke und die Simulation der Stimula- 


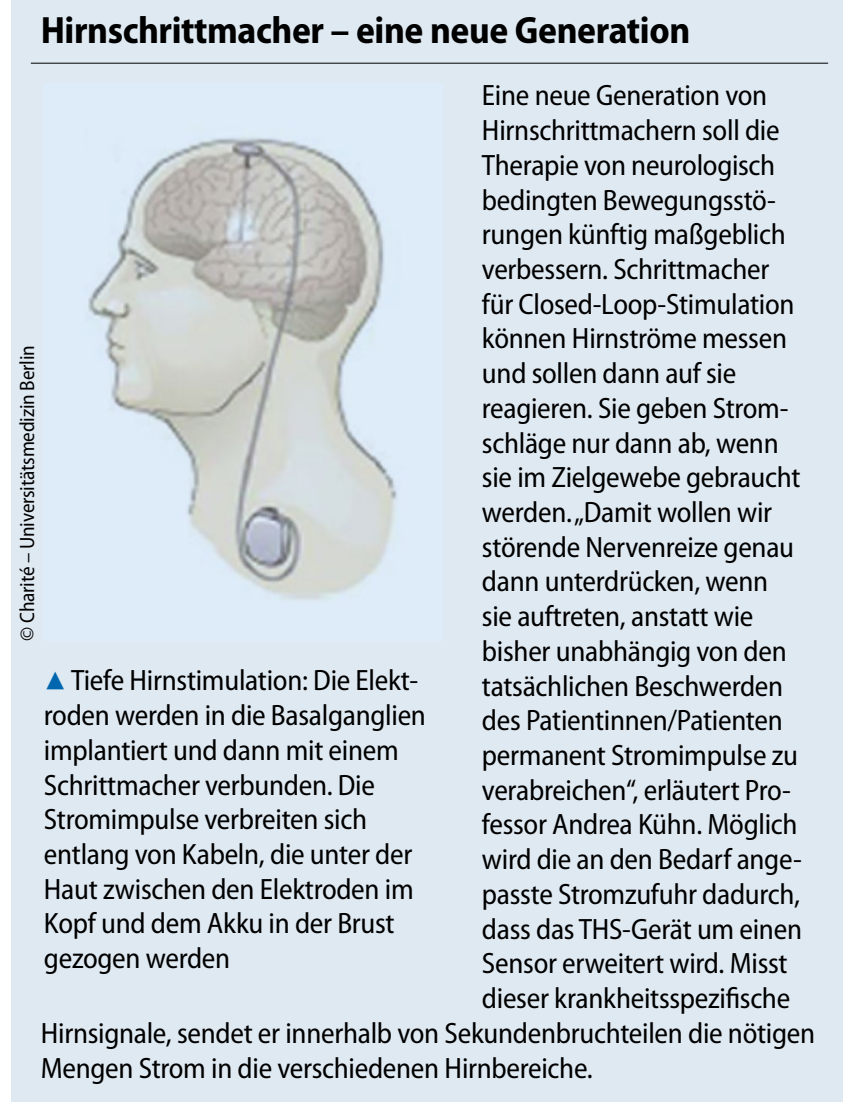

tionseffekte, die zur Therapieverbesserung beitragen können.

Konkret: Wie sehen die Arbeiten des SFB/Transregio der nächsten vier Jahre aus?

Für die Entwicklung bedarfsgesteuerter Schrittmachersysteme sollen charakteristische Hirnsignale bei normalen und krankhaft veränderten Bewegungsabläufen entschlüsselt und gezielt beeinflusst werden. Darüber hinaus untersuchen wir grundlegende Mechanismen der Hirnstimulation an Modellsystemen für Bewegungsstörungen, in tierexperimentellen Studien sollen neue Therapieansätze für Gangstörungen entwickelt werden. Diese klinischen und experimentellen Befunde werden in Computermodelle einfließen, mit deren Hilfe wir künftig individuell optimierte Stimulationsalgorithmen vorhersagen können. Das ist aber noch $\mathrm{Zu}$ kunftsmusik.
tinnen/Patienten vielleicht in $\mathrm{Zu}$ kunft profitieren können.

\section{Pionier der Teleneurologie}

Lee Schwamm hat eine Mission: Der Professor für Neurologie an der Harvard Medical School und Direktor des Comprehensive Stroke Center und Center for TeleHealth am Massachusetts General Hospital, Boston, USA, will entlegene Krankenhäuser mit neurologischer Expertise versorgen, die Art der medizinischen Versorgung revolutionieren und dem Gesundheitssystem viel Geld sparen. Sein Werkzeug: Teleneurologie.

Um 21.50 Uhr hat ein Mann, der sich zu Hause entspannt, plötzlich starke Kopfschmerzen und seine Worte verwischen. Um $22.42 \mathrm{Uhr}$ wird er in die Notaufnahme des örtlichen Krankenhauses gebracht, das Team diagnostiziert einen Schlaganfall. Um 22.55 Uhr erhält der Patient einen CT-Scan. Dann ruft die Ärztin/der Arzt vor Ort das TeleStroke-Center des Massachusetts General Hospital an.

Im Jahr 1996 hat der junge Neurologe Lee H. Schwamm gerade erst seine Facharztausbildung abgeschlossen, als er am Massachusetts General Hospital (MGH) im US-amerikanischen Boston das Projekt TeleStroke initiiert: Schlaganfall-Expertinnen/-Experten des MGH sollen sich per Video und Telefon mit Ärztinnen/Ärzten an Regionalkrankenhäusern im Nordosten der USA zusammenschalten, um diese in Echtzeit bei der Behandlung des akuten Schlaganfalls zu unterstützen.

Um 23.02 Uhr befindet sich der lokale Arzt in einer Videokonferenz mit dem Schlaganfall-Spezialisten des Massachusetts General Hospital. Zusammen führen sie eine kurze neurologische Untersuchung des Patienten durch. Krankengeschichte und aktuelle Medikation werden hochgeladen, und das Team diskutiert ihre Auswirkungen. Um 23.12 Uhr empfängt der Schlaganfall-Spezialist die Bilddateien des Gehirns und beginnt, sie auszuwerten.
Vielen regionalen und lokalen Krankenhäusern fehlen die finanziellen Mittel für eine 7/24-Schlaganfall-Versorgung. Die meisten verfügen nicht einmal über einen Neurologen. Schlaganfall gehört deshalb zu den vier häufigsten Todesursachen in den USA und ist die häufigste Ursache für vermeidbare Behinderungen. Lee $\mathrm{H}$. Schwamm: „Die traurige Wahrheit ist: Je abgelegener der Patient wohnt, desto geringer ist die Wahrscheinlichkeit, dass er nach einem Schlaganfall eine Thrombolyse erhält.“ Das MGH TeleStroke Center schafft Abhilfe: „Unsere Mission ist es, diesen Krankenhäusern die Expertise eines weltweit ausgewiesenen Schlaganfall-Zentrums zur Verfügung zu stellen", so Schwamm.

Um 23.15 Uhr steht die Diagnose fest: akuter ischämischer Schlaganfall, der den linken Frontallappen betrifft. Der Schlaganfall-Spezialist empfiehlt Thrombolyse, der Arzt vor Ort ordnet eine Behandlung an. 90 Minuten nach seinen ersten Symptomen erhält der Patient um 23.20 Uhr die Infusion.

Ende der 90er Jahre reagieren die Leitungen vieler Regionalkrankenhäuser zunächst wenig begeistert auf Schwamms Unterstützungsangebot. Sie fürchten, Kontrolle und Einnahmen zu verlieren. Das Gegenteil tritt ein: Dank der telemedizinischen Beratung können $65 \%$ aller Schlaganfälle vor Ort behandelt werden. „Das bedeutet Mehreinnahmen von sechs Millionen US-Dollar für die lokalen Krankenkenhäuser", rechnet Lee Schwamm vor. Mit der Zeit stellen die lokalen Ärzteteams fest, dass die Bostoner Kollegen nicht übernehmen wollen, sondern ihre Expertise nur im Bedarfsfall und auf Abruf zur Verfügung stellen. „Wir sind das Sicherheitsnetz - nicht mehr und nicht weniger", bringt es Schwamm auf den Punkt. Wenn ein Krankenhaus dem Schlaganfall-Netzwerk beitritt, schulen die Mitarbeiter des TeleStroke-Centers die lokalen Ärzte, Administratoren und IT-Mitarbeiter. Das Netzwerk soll 


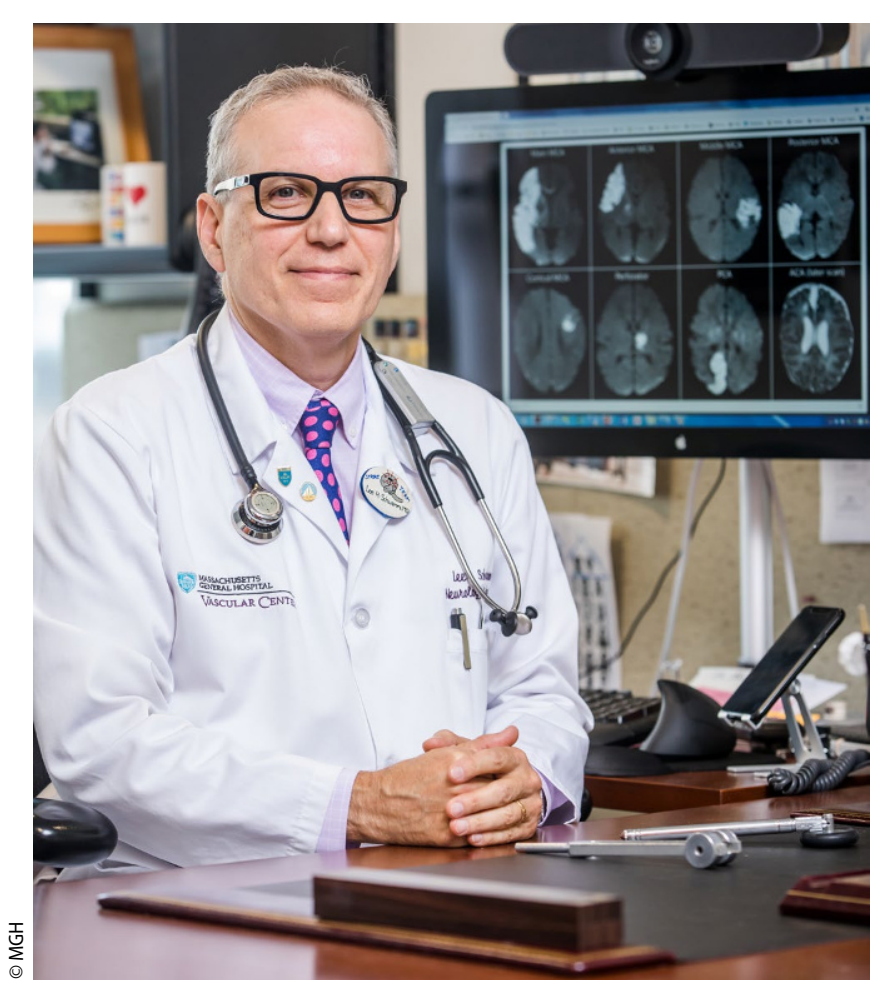

$\Delta$ Der Teleneurologie-Pionier Prof. Lee H. Schwamm ist Professor für Neurologie an der Harvard Medical School und leitet das Comprehensive Stroke Center und das Center for TeleHealth am Bostoner Massachusetts General Hospital, USA

reibungslos funktionieren, bevor der erste Patient eintrifft.

Heute haben mehr als 60 Notaufnahmen im Nordosten der USA rund um die Uhr und sieben Tage pro Woche Echtzeitzugriff auf Schlaganfall-Experten des MGH-TeleStroke-Netzwerks. Das Videokonferenzsystem, das Lee Schwamm als "Skype on Steroids" bezeichnet, bringt das Fachwissen der MGH-Mitarbeiter jederzeit und überall in die Notaufnahme. „Wir sind das größte kontinuierlich betriebene akademische TeleStroke-Zentrum des Landes, möglicherweise der Welt", sagt er stolz.

Und: Das Prinzip ,TeleStroke“ findet überall in den USA Nachahmer. Inzwischen verbinden 56 Netzwerke in 27 Bundesstaaten je ein Schlaganfall-Zentrum mit regionalen Krankenhäusern - mit direkten Auswirkungen auf die Überlebensrate: „Deutlich mehr Schlaganfall-Patienten erhalten eine lebensrettende Lyse-Therapie. In unserem Netzwerk sind es $40 \%$ der Schlaganfall-Patienten, zu verbessern. Telemedizinischer Versorgungsbedarf bestehe schließlich auch bei anderen neurologischen Erkrankungen. Schwamm und sein TeleHealthTeam bieten deshalb seit einigen Jahren neurologische Beratung und Interpretation zu fast allen neurologischen Krankheiten, sei es in der Notaufnahme oder beim Patienten zu Hause. Schwamm ist überzeugt: „Die Teleneurologie hat das Potenzial, die medizinische Versorgung zu revolutionieren: indem sie Unterschiede beim Zugang zu neurologischem Fachwissen beseitigt, die Zeit bis zur Bewertung für den Patienten verkürzt und dem Gesundheitssystem Kosten spart.“

Interview und Text: Monika Holthoff-Stenger

\section{Medizin trifft Musik: Konzert des Deutschen Neuro- Orchesters}

Das Deutsche Neuro-Orchester spielt am Dienstag, den 3. November 2020, um 20.00 Uhr. Das Konzert wird live auf dem Kongressportal ausgestrahlt. Eine Aufzeichnung des Konzerts steht anschließend zur Verfügung und kann z. B. als Ausklang eines virtuellen Kongresstags genossen werden.

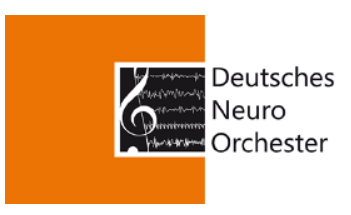

Auch in diesem Jahr wird es trotz widriger Umstände ein Konzert des Deutschen Neuro-Orchesters e. V. im Rahmen des virtuellen DGN-Kongresses geben!

Das Deutsche Neuro-Orchester wurde 2017 von Muriel Stoppe, Geigerin und Neurologin aus Leipzig, anlässlich des 90. DGNKongresses in Leipzig gegründet. Seitdem fanden 2018 und 2019 weitere erfolgreiche Konzerte im Berliner Konzerthaus und in der Stuttgarter Liederhalle statt. Das Ensemble besteht aus etwa 50 semiprofessionellen und Lai- enmusikern, die beruflich in der Neurologie und angrenzenden Bereichen der Medizin tätig sind.

Neben dem gemeinsamen Interesse an der Musik verbindet die Musikerinnen und Musiker des Orchesters der Wunsch, Initiativen aus dem Bereich der Neurologie durch Spenden zu unterstützen. Die Spendenerlöse aus den bisherigen Konzerten (mehr als 30.000 Euro) kamen u. a. Projekten aus den Bereichen Multiple Sklerose und Parkinson-Erkrankung zugute.

Im Dezember 2019 gründeten die Orchestermitglieder den gemeinnützigen Verein „Deutsches Neuro-Orchester e.V.“ (DeNO e. V.), um dem zunehmenden organisatorischen Aufwand gerecht werden zu können. Der Vereinsvorstand besteht aus Prof. Dr. Georg Gahn, Karlsruhe (1. Vorsitzender), Dr. Thorsten Süß, Berlin (2. Vorsitzender) und Dr. Huberta Engelhardt, Berlin (Schatzmeisterin).

Auch für den 93. DGN-Kongress 2020 war ein Konzert im Berliner Konzerthaus geplant, für das als besonderer Höhepunkt Alice Sara Ott, Pianistin

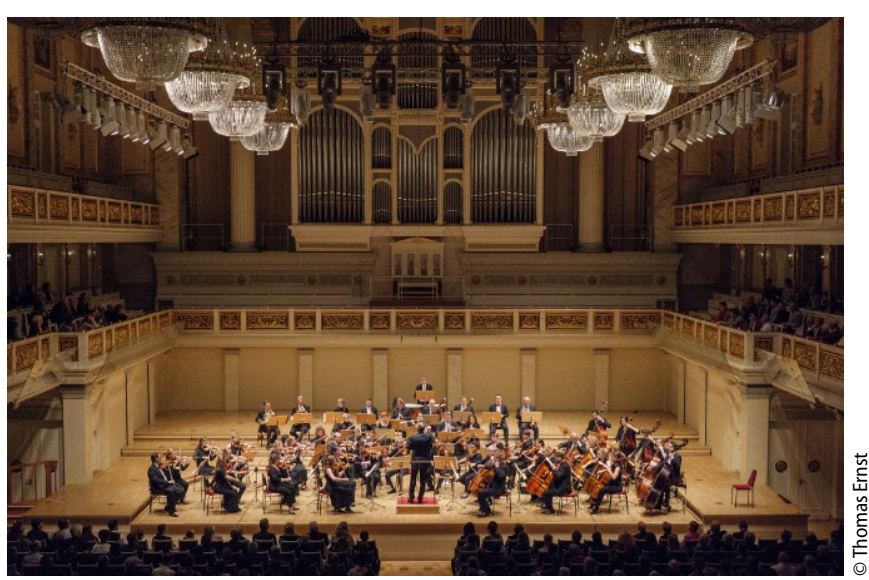

\ Während der Neurowoche 2018 spielte das Neuro-Orchester im Konzerthaus Berlin vor mehr als 1200 Zuhörern. Insgesamt konnten die Musiker mehr als 20.000 Euro für die unabhängige Multiple-Sklerose-Forschung sammeln 
von Weltrang, ihre Mitwirkung zugesagt hatte. Trotz der Enttäuschung über die Absage dieses Konzertes bestand innerhalb des Orchesters durchweg der große Wunsch, die Tradition eines Auftritts im Rahmen des DGN-Kongresses auch unter veränderten Bedingungen aufrechtzuerhalten. Den Orchestermitgliedern ist es besonders wichtig, gerade in diesen Zeiten den Benefizgedanken zu erhalten und zur Erfreuung der Kolleginnen und Kollegen und für die Unterstützung neurologischer Patientinnen/Patienten zu musizieren.

In kleinerer und den Hygienevorgaben angepasster Besetzung wird das Orchester unter der bewährten Leitung von Prof. Dr. Stefan Willich in diesem Jahr die Serenade für Streicher in CDur, Opus 48, von Pjotr Iljitsch Tschaikowski aufführen. Die Position der Konzertmeisterin wird dankenswerterweise von Daniela Braun-Friedrich vom Orchester der Komischen Oper Berlin übernommen.

Das Konzert wird am Vorabend des Kongresses, also am 3. November 2020, um 20.00 Uhr auf dem Kongressportal ausgestrahlt. Eine Aufzeichnung des Konzerts steht danach auf dem Portal zur Verfügung und kann z. B. als Ausklang eines virtuellen Kongresstags genossen werden.

Auch einen Spendenaufruf soll es in diesem Jahr geben der Spendenertrag soll der Deutschen Multiple Sklerose Gesellschaft Bundesverband e.V. und der Berliner Schlaganfall-Allianz (BSA e. V.) zugutekommen.

Bereits jetzt freuen wir uns auf unser nächstes Live-Konzert im Rahmen des DGN-Kongresses 2021 und hoffen, Ihnen auch mit dem diesjährigen virtuellen Konzert eine Freude zu bereiten.

Das Deutsche Neuro-Orchester ist der Gemeinnützigen Hertie-Stiftung auch in diesem Jahr für die großzügige Unterstützung zu herzlichem Dank verpflichtet, ohne die das Konzert nicht möglich wäre.

Weiterer Dank gebührt allen beteiligten Gremien der DGN sowie Prof. Dr. K. Toyka für die tatkräftige organisatorische Hilfe und der evangelischen Kirchengemeinde Schlachtensee für die Räume.

Mit herzlichen musikalischen und kollegialen Grüßen,

Ihre Georg Gahn, Thorsten Süß und Huberta Engelhardt

\section{Prof. Dr. Stefan N. Willich (Dirigent)}

Stefan Willich studierte Geige, Kammermusik und Dirigieren in Stuttgart und Berlin und nahm an renommierten Dirigierworkshops mit Sergiu Celibidache (München), Leon Fleisher (Boston, Tanglewood) und Leon Barzin (Paris) teil.

Sein beruflicher Werdegang führte ihn jedoch zunächst in die Medizin, wo er zu einem angesehenen Kardiologen und Forscher mit den Schwerpunkten HerzKreislauf-Erkrankungen, Prävention, Gesundheitsökonomie, integrative Medizin sowie Musik und Medizin wurde. Er arbeitete mehrere Jahre an der Harvard Medical School in Boston und wurde dann zum Professor und Direktor des Instituts für Sozialmedizin, Epidemiologie und Gesundheitsökonomie an der Charité - Universitätsmedizin Berlin berufen.

Von 2012 bis 2014 war Stefan Willich Präsident der Musikhochschule Hanns Eisler in Berlin.

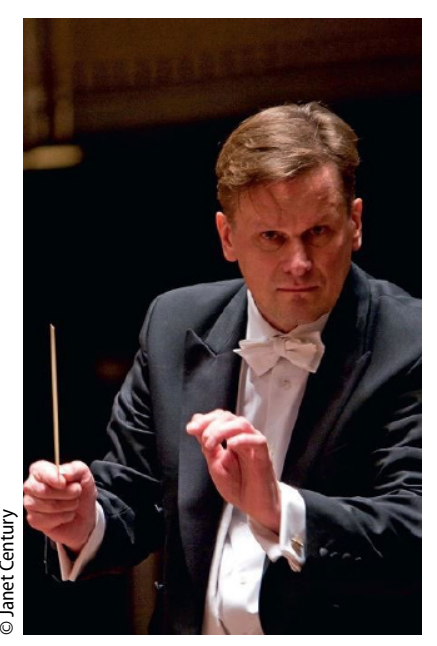

Stefan Willich tritt regelmäßig als Dirigent vor allem in Deutschland, Italien und den USA auf, wo er erster Gastdirigent des CityMusic Cleveland Orchestra ist. Im Jahr 2008 gründete er das World Doctors Orchestra (WDO), das seither weltweit viele Benefizkonzerte gegeben hat. Als künstlerischer Leiter führte er das WDO zu großem Erfolg. Besondere Höhepunkte waren die Aufführungen von Beethovens 9. Sinfonie in Berlin im Jahr 2010 anlässlich des World Health Summit sowie Mahlers 2. Sinfonie in Washington im Jahr 2011 anlässlich des 10. Jahrestages des 11. September. Stefan Willich arbeitete mit herausragenden Solisten wie Peter Zazofsky, Sergey Khachatryan, Tanja Becker-Bender, Aida-Carmen Soanea, Tamaki Kawakubo, Wenzel Fuchs, Anja Kampe, Jeanine De Bique, Falk Struckmann, Jochen Kowalski, Alexei Lubimov, Ludwig Quandt und mit dem Wiener Knabenchor.

\section{„Neurologen und Neurowissenschaftler in der NS-Zeit": Leserstimmen}

2015 hat die DGN ein auf sechs Jahre angelegtes Forschungsprojekt angestoßen und renommierte Me-

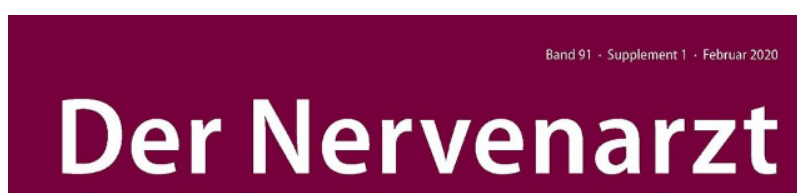
Organ der Deutschen Gesellschaft für Psychiatrie und Psychotherapie, Psychosomatik und
der Deutschen Gesellschaft fur Neurologie und der Deutschen Schlaganfall-Gesellschaft

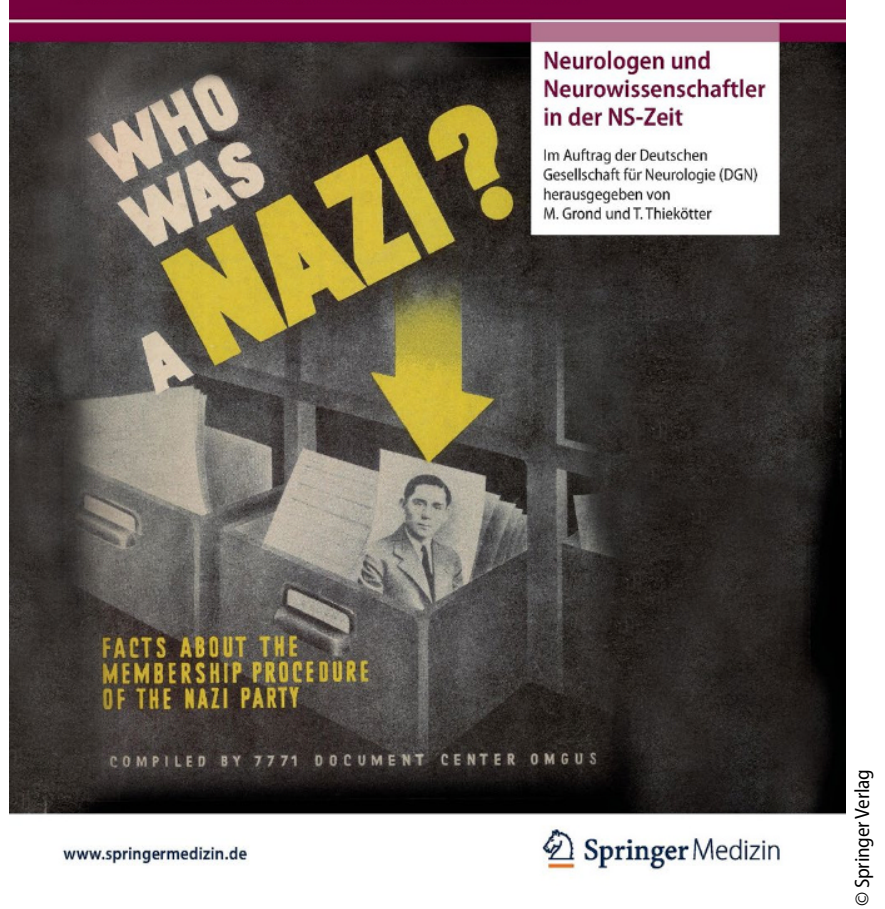

$\Delta$ Cover des Supplements "Neurologen und Neurowissenschaftler in der NS-Zeit" 
mitglieder in das NS-Regime aufzuarbeiten: Prof. Axel Karenberg (Köln), Prof. Heiner Fangerau und Dr. Michael Martin (Düsseldorf) von den jeweiligen Instituten für Geschichte und Ethik der Medizin dieser Universitäten haben eine umfassende Publikation vorgelegt, die im Februar als Supplement in „Der Nervenarzt" ausgeliefert wurde.

Erfreulicherweise hat das Supplement $\mathrm{zu}$ einem lebendigen Diskurs innerhalb der DGN geführt - in der Juni-Ausgabe von „Der Nervenarzt“ wurden verschiedene Leserbriefe von Mitgliedern veröffentlicht, die eine ganze Bandbreite an Reaktionen zeigen: von erleichterten Beiträgen - „endlich eine längst überfällige Aufarbeitung “ - über einen Rehabilitationsversuch eines von Historikern als belastet eingestuften Neurologen bis hin zu Stimmen, die Parallelen zur heutigen Situation aufzeigen, in der Ärztinnen und Ärzte auch nicht immer nach bestem Wissen und Gewissen handeln könnten: Zwar unterlägen sie keinen politischen, aber gravierenden, die ärztliche Freiheit limitierenden ökonomischen Zwängen. Weitere Leserstimmen sind eingetroffen, die interessante Denkanstöße geben und die wir Ihnen hier vorstellen möchten.

\section{„Ich habe es damals sehr bedauert, dass die DGPPN und die DGN diesen Weg der Aufarbeitung nicht gemeinsam gegangen sind"}

\section{Psychiatrie - Neurologie - Nervenheilkunde}

\section{Prof. Dr. Dr. Frank Schneider, Düsseldorf, federführender Herausgeber "Der Nervenarzt"}

Es ist wichtig, dass die DGN sich mit ihrer eigenen Vergangenheit in der Zeit des Nationalsozialismus beschäftigt hat [1]. Dafür gelten ihr Respekt und Anerkennung.

Nachdem Werner Hacke als neurologischer Schriftleiter dieser Zeitschrift die Initiative kommen-

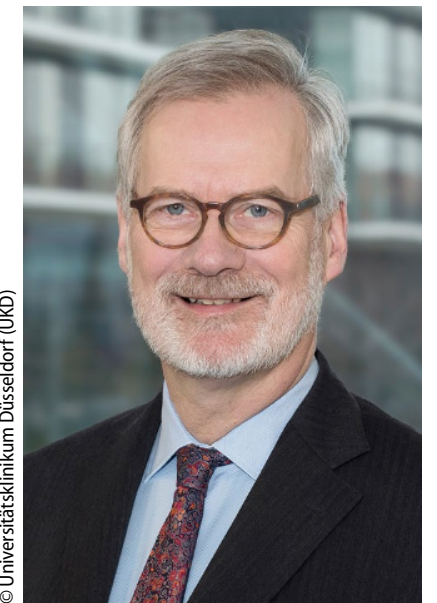

den ersten Nachkriegsjahrzehnten vermissen wir Mitgefühl mit den Opfern von Zwangssterilisierungen und Patiententötungen, inhumaner Forschung und den zwangsweise emigrierten jüdischen und politisch verfolgten Kolleginnen und Kollegen. Eine ernsthafte Aufarbeitung der Geschichte des eigenen Fachs im Nationalsozialismus begann seitens der Psychiater erst nach Jahrzehnten, viel zu spät.

Einzelne Psychiater wie zum Beispiel Gerhard Schmidt direkt nach dem Kriegsende oder Klaus Dörner in den 1960er Jahren und später Michael von Cranach oder Asmus Finzen, versuchten, das Geschehene aufzuarbeiten und bekannt zu machen. Diese Ansätze wurden aber in der Fachgesellschaft nicht nachhaltig gehört. Eine Ausnahme bildete das Jahr 1986, als die Deutsche Gesellschaft für Psychiatrie und Nervenheilkunde Gerhard Schmidt mit der Wilhelm-Griesinger-Medaille ehrte [3].

Im Jahr 1999 wurde in Hamburg auf dem Kongress der World Psychiatric Association (WPA) erstmals die Ausstellung von Michael von Cranach „In Memoriam" über die Verbrechen an Patientinnen und Patienten im Nationalsozialismus einem breiten internationalen Publikum zugänglich gemacht. Im Rahmen des Jahreskongresses der psychiatrischen Fachgesellschaft (damals noch als DGPN bezeichnet) im Jahr 1992 wurde eine Resolution verabschiedet, in der die Gesellschaft „ihren Abscheu und ihre Trauer im Rückblick auf den Holocaust an Geisteskranken, Juden und anderen verfolgten Menschen“ bekräftigte. Dies waren sehr deutliche, aber auch notwendige Worte, auch wenn es hier noch nicht um persönliche oder institutionelle Schuld von Psychiatern oder deren Fachgesellschaft ging. Nachfolgend arbeiteten einzelne Kliniken und Institutionen ihre Geschichte und Verstrickungen in der Zeit des Nationalsozialismus auf.

Im Jahr 2009 begann die DGPPN, sich sehr systematisch mit der eigenen Geschichte auseinanderzusetzen. Auf einer Mitgliederversammlung in diesem Jahr wurde der erste Paragraph der Satzung um folgenden Abschnitt ergänzt: „Die DGPPN ist sich ihrer besonderen Verantwortung um die Würde und Rechte der psychisch Kranken bewusst, die ihr aus der Beteiligung ihrer Vorläuferorganisationen an den Verbrechen des Nationalsozialismus, an massenhaften Krankenmorden und Zwangssterilisationen erwachsen."

Ich selbst habe es damals sehr bedauert, dass die DGPPN und die DGN diesen Weg nicht gemeinsam gegangen sind, obwohl die historischen Gegebenheiten dies nahegelegt hätten. Da eine Organisation zur Aufarbeitung der eigenen Geschichte aus verschiedenen Gründen selbst nicht geeignet ist, hat der seinerzeitige DGPPN-Vorstand 2009 eine internationale Kommission von Historiker/-innen zur Aufarbeitung der Geschichte der Vorläufergesellschaften zur Zeit des Nationalsozialismus eingerichtet. Die Kommission war in ihren Entscheidungen immer unabhängig von der DGPPN, um vollständige Transparenz zu gewährleisten. Sie begleitete die von der Fachgesellschaft initiierten und finanzierten Forschungsprojekte, die klären sollten, inwieweit die Vorläuferorganisationen und deren Repräsentanten an dem Euthanasie-Programm, an der Zwangssterilisierung, an der Vertreibung jüdischer und politisch missliebiger Psychiater und an anderen Verbrechen in der Zeit zwischen 1933 bis 1945 beteiligt waren.

Die DGPPN hat im Jahr 2009 beschlossen, dass der jeweilige Präsident der Gesellschaft den seinerzeit vergebenen Hermann-Simon-Preis nicht mehr durch seine Teilnahme im Kuratorium unterstützt. Hintergrund war die nationalsozialistische Verstrickung von Simon. Dann folgte die Unterstützung zur Errichtung eines Denkmals zur Erinnerung an die Morde an psychisch und neurologisch Kranken in Mogilew. 
Eine Gedenkveranstaltung mit über 3000 Teilnehmern in Berlin zur Erinnerung und Verpflichtung führte die DGPPN 2010 in Berlin durch [4]. Viele weitere Aktivitäten folgten, insbesondere eine von inzwischen über 500.000 Menschen auf allen Kontinenten besuchte Ausstellung ,erfasst, verfolgt, vernichtet: Kranke und behinderte Menschen im Nationalsozialismus" $[5,6]$.

Als Ergebnis des ersten Forschungsauftrages der DGPPN hat schließlich Hans-Walter Schmuhl im Jahr 2016 die Monographie „Die Gesellschaft Deutscher Neurologen und Psychiater im Nationalsozialismus" vorgelegt [7]. In ihr wurde belegt, dass das Netzwerk um den Vorsitzenden der Gesellschaft Deutscher Neurologen und Psychiater (GDNP), Ernst Rüdin, bei der praktischen Umsetzung des „Gesetzes zur Verhütung erbkranken Nachwuchses", d.h. bei der massenhaften zwangsweisen Sterilisierung vermeintlich „erbkranker" Menschen, eine Schlüsselrolle spielte. Es funktionierte die Zusammenarbeit zwischen der damaligen Fachgesellschaft und der „T4“Zentrale, also der bei der sogenannten Euthanasie federführenden Stelle, reibungslos. Zentrale Akteure der GDNP befürworteten die Patiententötungen nicht nur, sondern unterstützten das Vorgehen gegen vermeintlich ,wertloses" Leben auch in der Praxis. Es ist festzustellen, dass zahlreiche Psychiater und Neurologen, die in der Fachgesellschaft hohes Ansehen genossen und in ihr zum Teil auch führende Posten besetzten, eine Mitschuld an der Unfruchtbarmachung von bis $\mathrm{zu} 400.000$ Menschen und am Mord von mehr als 200.000 Menschen in Heil- und Pflegeanstalten trugen.

Nach dem erfolgreichen $\mathrm{Ab}$ schluss dieses ersten Forschungsprojektes hat die DGPPN - wieder über eine unabhängige Historikerkommission - einen zweiten Forschungsauftrag zur Geschichte der Psychiatrie im Nachkriegsdeutschland ausgeschrieben. Nun sollten die lang anhaltenden Folgen der nationalsozialistischen Psych- iatriepolitik, der fachinterne Umgang mit den Medizinverbrechen, die Ursachen für den fortdauernden Reformstau und die Gründe für das Versanden von Reforminitiativen zur Verbesserung und Ausdifferenzierung der psychiatrischen Versorgungseinrichtungen geklärt werden. Steffen Dörre hat seine Monographie hierzu gerade vorgelegt [8]. Damit führt die DGPPN eines der Versprechen auf der Gedenkveranstaltung der DGPPN 2010 fort, aktiv die Geschichte ihrer Vorläuferorganisationen im Nationalsozialismus und in der Zeit danach zu erforschen. Sowohl Schmuhls Buch als auch das Projekt über die Nachkriegszeit von Dörre zeigen, wie schwierig es für die untersuchten Epochen ist, Neurologie und Psychiatrie klar voneinander zu separieren, wirkten Neurologen und Psychiater in ihrer Außendarstellung und Eigenwahrnehmung doch stets zumindest unter Bezugnahme aufeinander.

Viel zu spät haben wir Psychiater unsere Aufgabe der Ehrung der Opfer und die Aufarbeitung des begangenen Unrechts im $\mathrm{Na}$ tionalsozialismus begonnen. Dafür schämen wir uns. Die DGPPN ging in den letzten Jahren diesen Weg des Erinnerns und der Verantwortungsübernahme oft alleine, wunderbar, dass die neurologische Fachgesellschaft nun folgt. Wir haben gemeinsame Wurzeln und sollten diese nicht vergessen. Auseinandersetzungen über die fachliche Identität und Debatten über das Fortlebenlassen der gemeinsamen Zeitschrift gab es in der Geschichte der DGPPN und DGN auch in der Bundesrepublik Deutschland immer wieder, wie Dörre in seinem Buch über die DGPPN aufzeigt [8]. Diese gemeinsame Vergangenheit ist spannend und sollte in Erinnerung bleiben.

Aber wir haben auch eine gemeinsame Zukunft! Die Entscheidung des Vorstands der DGN, dass „Der Nervenarzt“ zum Ende des Jahres 2022 als Mitgliederjournal gekündigt wurde, sollte überdacht werden. Auch der heute 91. Jahrgang der Zeitschrift zeigt, dass beide Fächer eine eige- ne Identität haben, aber trotzdem eng zusammengehören.

\section{Literatur}

1. Grond M, Thiekötter T (2020) Neurologen und Neurowissenschaftler in der NS-Zeit. Nervenarzt 91(Suppl. 1):1-2. https://doi.org/10.1007/ s00115-016-0145-6

2. Hacke W (2020) Leserbrief. Nervenarzt 91:556-557

3. Schneider F (Hrsg). Schmidt G: Selektion in der Heilanstalt 1939-1945. Neuausgabe mit ergänzenden Texten. Springer, Heidelberg

4. Schneider F (2011) Psychiatrie im Nationalsozialismus. Erinnerung und Verantwortung/Psychiatry under National Socialism. Remembrance and Responsibility. Springer, Berlin

5. Schneider F, Lutz P (2014) erfasst, verfolgt, vernichtet: Kranke und behinderte Menschen im Nationalsozialismus/registered, persecuted, annihilated:The Sick and the Disabled under National Socialism. Springer, Heidelberg

6. Schneider F (2019) erfasst, verfolgt, vernichtet. Kranke und behinderte Menschen im Nationalsozialismus. Die Dokumentation zur Ausstellung. Springer, Heidelberg

7. Schmuhl HW (2016) Die Gesellschaft deutscher Neurologen und Psychiater im Nationalsozialismus. Springer, Heidelberg

8. Dörre S (im Druck) Zwischen NS„Euthanasie" und Reformaufbruch. Die psychiatrischen Fachgesellschaften im geteilten Deutschland (1945-1975). Springer, Heidelberg

\section{„Es handelt sich um eine sorgfältig recherchierte Studie, die mir neue Einblicke erlaubte - auch solche, die ich nicht wahr- haben wollte"}

\section{Prof. em. Dr. Volker Dietz, Zürich/Schweiz}

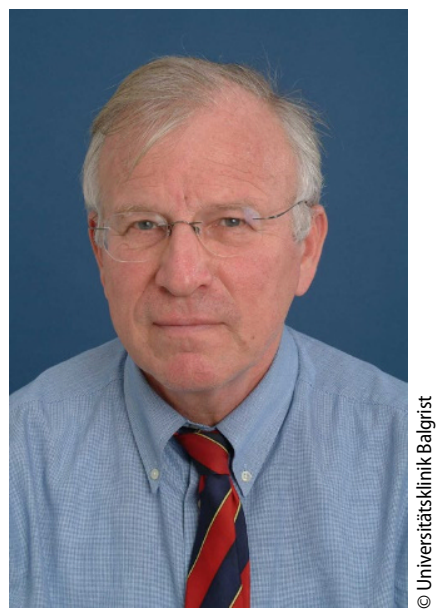

Ich habe das Heft über die „Neurologen und Neurowissenschaftler in der NS-Zeit" mit großem Interesse gelesen. Es waren meine Lehrer und wissenschaftlichen Vorbilder, die großen Einfluss auf mein damaliges klinisches und wissenschaftliches Denken hatten. Der Deutschen Gesellschaft für Neurologie sei Dank, dass sie diese Studie über die Verwicklungen von Neurologen und Neurowissenschaftlern in der NS-Zeit in Auftrag gegeben hat. Damit wurde diese Ära auch von neurologischer Seite her aufgearbeitet. Es handelt sich um eine sorgfältig recherchierte Studie, die mir, der sich persönlich (z. B. Rolle der Eltern) und klinisch/ wissenschaftlich (z.B. Euthanasie) intensiv mit der NS-Zeit befasst hatte, neue Einblicke erlaubte - auch solche, die ich nicht wahrhaben wollte.

So verdienstvoll diese Studie ist - welche Konsequenzen soll die heutige Neurologinnen/ Neurologen-Generation daraus ziehen und wie soll mit diesen Erkenntnissen umgegangen werden? Diese Überlegungen scheinen mir besonders im Hinblick auf die Namensgebung von Preisen und designierten Veranstaltungen wichtig. Werden die Verdienste um wichtige Weichenstellungen in der Neurologie und Errungenschaften in der Neurowissenschaft durch eine Mitgliedschaft in der NSDAP geschmälert? Sind spektakuläre Forschungserkenntnisse (soweit sie unter ethischen Voraussetzungen erlangt wurden) weniger wert aufgrund persönlichen Fehlverhaltens? Die Bedeutung der Philosophie Heideggers kann trotz seiner Nähe zum Nationalsozialismus nicht negiert werden - das sollte meines Erachtens auch für bahnbrechende neurowissenschaftliche Erkenntnisse gelten. Ich votiere für die Ehrung einer Persönlichkeit mit der Namensgebung von Preisen im Bereich Neurologie und Neurowissenschaft, die primär auf der Bedeutung der mit einem Namen verbundenen Erkenntnis beruht. In Zukunft sollten jedoch bei 
dem mit dem Preis verbundenen Namen nicht nur die Verdienste (wenn es solche tatsächlich auch im Nachhinein waren), sondern auch ein persönliches Fehlverhalten der Persönlichkeit festgehalten werden. Dazu ist eine sorgfältige Abwägung erforderlich. In Zukunft sollte vor neuen Würdigungen von Persönlichkeiten durch an Preise oder Veranstaltungen geknüpfte Namen zuvor etwaiges Fehlverhalten, wie antisemitische/rassistische Äußerungen, eruiert werden.

\section{„Das Supplement 'Who was a Nazi?' setzt einen Meilenstein für den Umgang mit ärztlichen Fehlentscheidungen"}

\section{Dr. Hannah Preßler, Berlin}

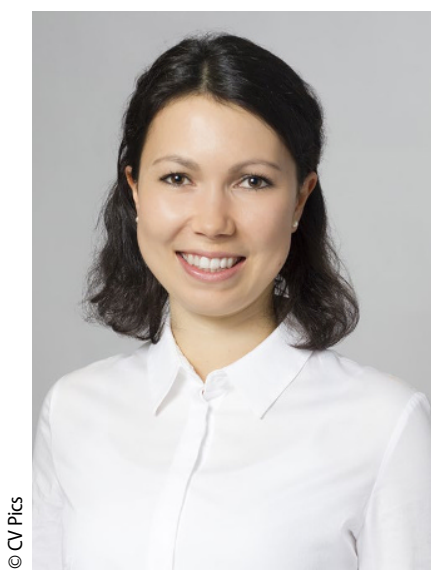

Die Berichte und Dokumentationen zeigen, wie vielschichtig und ambivalent der menschliche Geist war, ist und in Zukunft sein wird. Wir können Entscheidungen nicht für unsere Vergangenheit und auch nur begrenzt für unsere Zukunft treffen. Ebenso wenig wie Angst und Mut einen Widerspruch bilden, stehen Zivilcourage und menschliche Schwäche konträr zueinander. Bereits Aristoteles stellte im übertragenen Sinne fest, der Mensch ist mehr als die Summe seiner Teile. Jeder kritische Beitrag, jede Auseinandersetzung und jeder Fehler liefern wichtige Kenntnisse für eine Diskussion bezüglich zukünftiger Handlungsstrategien im verantwortungsbewussten und reflektierten Umgang mit unserer gemeinsamen Vergangenheit als Neurologen/Neurologinnen. Als Assistenzärztin am Jüdischen Krankenhaus Berlin wurde ich in der Einführungsveranstaltung für neue Mitarbeiter und Mitarbeiterinnen unmittelbar mit der besonderen Geschichte des Hauses im Nationalsozialismus und der damit einhergehenden Diskriminierung von Ärztinnen/ Ärzten und Patientinnen/Patienten vertraut gemacht. Die besondere Geschichte des eigenen Arbeitsplatzes zu kennen, sensibilisierte mich zugleich für das heutige Arbeiten hier in Berlin und die Entwicklung des Hauses nach der NS-Diktatur.

Das Supplement der Februarausgabe des „Nervenarztes“ 2020 „Who was a Nazi?" setzt damit einen Meilenstein für den Umgang mit ärztlichen Fehlentscheidungen. Wenn man sich mit den Gräueltaten des NS-Regimes befasst, offenbart sich der berühmte Ausdruck Hannah Arendts, „die Banalität des Bösen“, der besagt, dass sich das Böse durch seine Institutionalisierung $\mathrm{zu}$ etwas Banalem entwickelt. Folglich können wir dem Bösen nur widerstehen, wenn wir nachdenklich bleiben. Wir alle nutzen kognitive Vereinfachungen, um mit der Komplexität unserer Umwelt umzugehen, weil es das Gehirn wenig Energie kostet und so Ressourcen geschont werden. Diese Ressourcen werden aber in gesellschaftlichen Umbrüchen und wandelnden Systemen umso dringlicher, wenn automatisierte Handlungsabläufe letztlich nicht mehr zukunftsorientiert oder retrospektiv gesehen zu furchtbaren Fehlern führen.

Ein System, das keine Fehler macht, ist nicht menschlich und darüber hinaus auch nicht intelligent. Sich gegen eine defensive Medizin zu entscheiden, bedeutet auch, sich für eine positive Fehlerkultur einzusetzen.

Insbesondere für junge Ärztinnen und Ärzte wird nach Beendigung der Universität einmal mehr deutlich, dass sich unser Berufsbild in einem Wertewandel vom Heiler der alten Welt zum Arzt einer neuen Generation entwickelt. Zum einen hält die moderne Medizin viele neue diagnostische und therapeutische Instrumente für unsere alternde Gesellschaft bereit, zum anderen erfordert sie für die praktische Umsetzung deutlich mehr finanzielle und personelle Ressourcen. Viele Ärztinnen und Ärzte haben sich aus Angst, selbst Opfer der Nazi-Diktatur zu werden, deren Gesetzmäßigkeiten untergeordnet. Wenn wir, wenn auch in einer ganz anderen Dimension, nicht erneut einer paternalistischen Medizin unterliegen möchten, die uns zu defensiven Entscheidungen zwingt, müssen wir nachdenklich bleiben, Verantwortung übernehmen und Risikokompetenz für unsere künftigen Herausforderungen der modernen Medizin erwerben.

Damit wir uns und unsere $\mathrm{Pa}$ tientinnen/Patienten im 21. Jahrhundert nicht in eine Opferrolle begeben, ist es erneut an der Zeit, sich proaktiv gegen paternalistische Medizin aufzulehnen, gegen Manager/Managerinnen, Politiker/Politikerinnen, Krankenkassen, die Wirtschaftlichkeitskriterien über unsere Verantwortung stellen, moderner und zukunftsorientierter Medizin ein moralisch vertretbares Gesicht zu geben. Als Arzt oder Ärztin im 21. Jahrhundert gehört es zu unserer Pflicht, genauso visionär, ambitioniert und sinnorientiert für unser Gesundheitswesen einzustehen wie für jeden einzelnen Patienten, den wir behandeln dürfen. So wie wir in unserem Fachgebiet als Spezialisten/Spezialistinnen agieren, so müssen wir im Hinblick auf unser sich wandelndes Gesundheitssystem als Generalisten/Generalistinnen handeln und denken.

\section{"Wichtig wäre die Frage, was heute eine gute Ärztin/einen guten Arzt ausmacht"}

\section{Dr. Margrit Eysholdt, Hildes-} heim

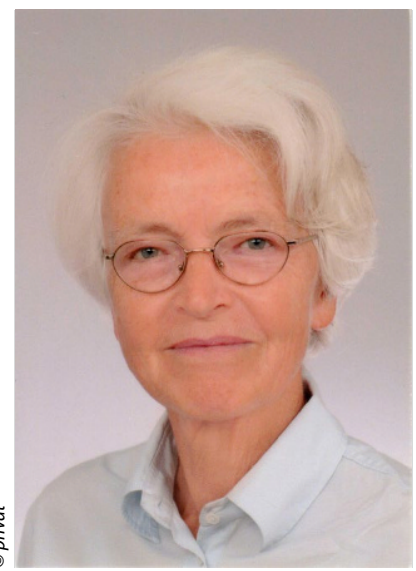

Das Supplement zum „Nervenarzt" vom Februar 2020 fand ich in besonderem Maße interessant und lesenswert. Unser Geschichtsunterricht in der Schule endete mit dem Jahr 1933. Ich finde gut, über diese Dinge so offen wie möglich zu reden, ohne zu verurteilen. Jede Arztpersönlichkeit wird anfangs durch Erziehung geprägt. Und ich denke, dass die NS-Erziehung bis in das dritte und vierte Glied fortwirkt. Früher fand ich uns Deutsche zu leistungsbezogen bei einem ungesicherten Selbstwertgefühl. Das hat sich zwischenzeitlich, glaube ich, gebessert. Wichtig wäre die Frage, was heute eine gute Ärztin/einen guten Arzt ausmacht. Ich denke, jeder Kollege hat dazu irgendwelche Vorstellungen, aber die gehen weit auseinander. Was ökonomische Zwänge angeht, so haben zumindest niedergelassene Ärztinnen/ Ärzte bei einem durchschnittlichen Jahreseinkommen von 180.000 Euro Spiel nach unten. Angesichts der Empfehlungen von Big Pharma und Banken bei der Niederlassung wünsche ich jedem Kollegen Rückgrat. 


\section{"Welche Konsequenzen ziehen wir aus der Vergan- genheit?"}

\section{Dr. Claudia Busch, Fachärztin für Neurologie}

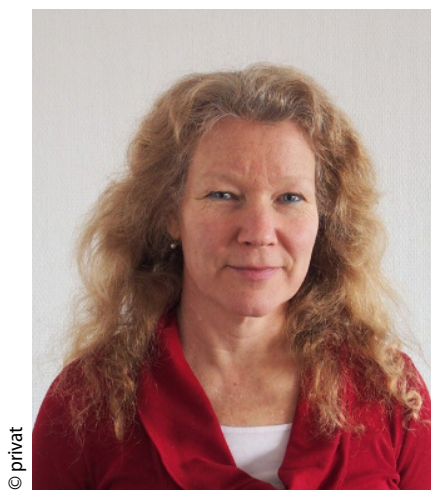

Ich freue mich sehr über die Bemühungen einer Aufarbeitung und die rege Diskussion, die sich angeschlossen hat.

Ich bin in der eigenartigen Situation, dass meine medizinischen Vorfahren eine meiner familiären Vorfahrinnen (die Schwester meiner Mutter, siehe auch https://www.t4-denkmal.de/Wilhelmine-Haussner) umgebracht haben, daher bin ich in dieser Diskussion alles andere als unvoreingenommen.

Von der Bildunterschrift der Illustration auf der Titelseite „Who was a Nazi?" war ich erst mal ziemlich irritiert, wieso „Nazi“? Die Überlegung, wie weit der einzelne Arzt infiziert vom nationalsozialistischen Gedankengut war oder welche Punkte er kritischer sah, ist ja noch schwerer zu rekonstruieren als die Taten. Außerdem haben sich nicht die "Nazis“ die sogenannte Euthanasie ausgedacht, sondern unsere ärztlichen Vorfahren schon vor der NaziZeit. Das ist, was mich besonders quält, die menschenverachtende Haltung Patientinnen/Patienten und Schutzbefohlenen gegenüber. Das ist in dem militaristischen Schwarz-Weiß-Denken des Dritten Reichs auf fruchtbaren Boden gefallen und hat auch die Möglichkeiten des Forschens deutlich erweitert. Hat man den Menschen erst ihre Menschenwürde genommen, lässt sich an allen anderen Menschenrechten auch sehr schnell einsparen, schon war man bei „lebensunwert“.

Sehr vorsichtig finde ich die Annäherung dafür an die $\mathrm{Ge}$ schichte und unsere ehrenwerten neurologischen Forscher und Vorfahren. Sie haben folgenschwere Entscheidungen getroffen, die auf Fehleinschätzungen der menschlichen Würde basierten, das darf gedacht und laut gesagt werden und wurde von Medizinhistorikern wie z.B. Gerrit Hohendorf schon seit Jahrzehnten sehr genau untersucht. Auch in den damaligen Heil- und Pflegeanstalten gibt es eine breite Aufarbeitung und Aufklärung der verhängnisvollen Entwicklung.

Was können wir daraus lernen? Welche Konsequenzen ziehen wir aus der Vergangenheit?

Für mich ist es sehr wichtig, meine Patientinnen/Patienten nach Möglichkeit in ihrer Persönlichkeit anzunehmen und sie nicht nur als Fall möglichst schnell abzuhandeln. Eine gute Neurologie besteht, wie unsere Ziehväter auch wussten, aus einer guten Anamnese und der Fähigkeit, aufmerksam und auch selbstkritisch zuzuhören. $\mathrm{Zu}$ diskutieren wäre auch eine höhere Wertschätzung der sprechenden Medizin mit angemessener Honorierung.

Äußerste Vorsicht ist geboten, wenn wir im besten Sinne meinen, für andere Entscheidungen treffen zu können.

Ich bin sehr gespannt auf die weitere Diskussion!

\section{„Was haben wir noch mit der Nazi-Zeit zu tun?"}

\section{Pieter Rodgers, Facharzt für Neurologie, Hilden}

Was soll das ... ? Was haben wir noch mit der Nazi-Zeit zu tun? Nichts ... - und ich als niederländischer Arzt (Neurologe) in Deutschland noch viel weniger ... Gibt's noch deutsche Ärztinnen/ Ärzte, die daran beteiligt waren, die derzeit noch leben? Vielleicht gibt es noch wenige Patientinnen/ Patienten, aber Ärztinnen/Ärzte aus dieser Zeit dürfte es doch kaum noch geben. Großes Problem ist auch, dass viele Ärztin-

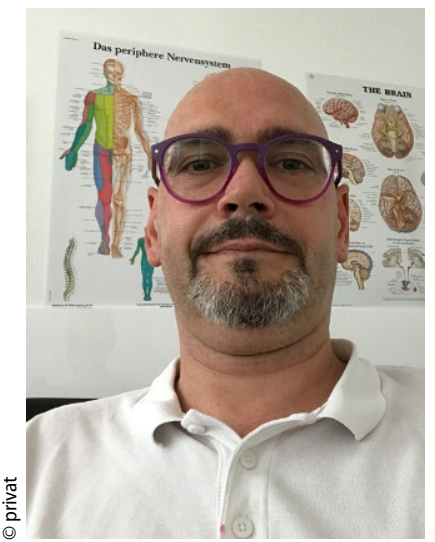

nen/Ärzte und auch Richter, die aktiv mitgeholfen haben in dieser Zeit, nach dem Krieg nicht verfolgt oder ihres Amtes enthoben wurden: Wo holt man auch so schnell neue Ärztinnen/Ärzte und Richterinnen/Richter her?

Ihr Deutschen habt die Nazi-Zeit schon längst verarbeiten können, wie dies die restlichen Länder in Europa auch getan haben. Die derzeitige Generation hat damit nichts mehr am Hut!

\section{Vorgestellt: DGN- Kommissionen}

Die Deutsche Gesellschaft für Neurologie (DGN) hat ihre Kommissionen neu geordnet. Mit der Ausgabe 10/2019 des "Der Nervenarzt" startete die Reihe „DGN-Kommissionen", mit der wir in jeder Ausgabe neue und neu besetzte Kommissionen kurzen Interviews mit den Kommissionssprechern vorstellen. Heute stellen wir Ihnen die Kommission „Prävention in der Neurologie" vor und sprachen mit Kommissionsleiter Prof. Dr. Stefan Isenmann, Moers.

\section{Kommission 2.13 Präven- tion in der Neurologie}

„Wir möchten das Wissen zu Prävention zusammentragen und dazu beitragen, dass präventive Maßnahmen gesundheitsfördernd umgesetzt werden!"

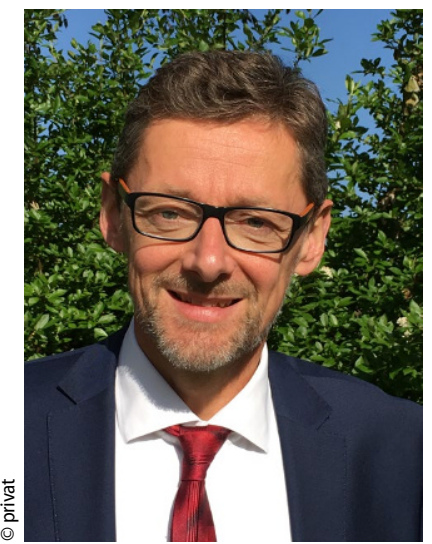

Welche Aspekte umfasst die „Prävention in der Neurologie"?

Die Sozialmediziner unterscheiden grundsätzlich drei Stufen der Prävention: zunächst die generelle Vorbeugung von Erkrankungen, also das, was im Volksmund ganz allgemein unter Prävention verstanden wird. Im neurologischen Bereich sind da die beiden großen Indikationen Schlaganfall und Demenz zu nennen. Es gibt eine hohe Evidenz, dass Bewegung und Ausdauersport dem Auftreten von Schlaganfällen vorbeugen. Auch gibt es Daten, die zeigen, dass Bewegung die Entwicklung einer Demenzerkrankung zumindest verlangsamen kann. Die WHO empfiehlt, fünfmal pro Woche 30 Minuten Sport zu treiben. Aber neben Sport und Bewegung zählen natürlich auch andere Faktoren zur Primärprävention. Bei der Demenz z. B. spielen Lebensführung, also Ernährung, kognitive Aktivität (lesen, denken, sich unterhalten, debattieren), Schlaf und natürlich auch ein gewisses Vermeidungsverhalten, also nicht zu rauchen, nicht viel fernzusehen etc., ebenfalls eine große Rolle.

Primärprävention betrifft alle Menschen. Der wissenschaftliche Zugang zu Primärprävention ist methodisch aufwendig und schwierig: Vieles kann man kaum 
nach strengen wissenschaftlichen Kriterien (prospektiv, randomisiert, multizentrisch, doppelt verblindet) langfristig untersuchen - stattdessen ist man auf indirekte Evidenz, auf Schlüsse angewiesen. So gesteht selbst die WHO ein, dass ihre Empfehlungen zu regelmäßiger körperlicher Aktivität zur Krankheitsprävention eher gesundem Menschenverstand und Erfahrungswissen entspringen als solider wissenschaftlicher Evidenz. Gleichzeitig ist es auch schwierig, Erkenntnisse zur Prävention durchzusetzen und im allgemeinen Bewusstsein als Notwendigkeit, ja Selbstverständlichkeit im Alltag eines jeden zu verankern. Dennoch: Daran müssen wir arbeiten, gerade auch wir Neurologinnen/Neurologen.

Bei der Sekundärprävention wird es deutlich konkreter und oft ist auch die "Compliance" gegeben. Nach einem Schlaganfall hat man in der Regel ein hohes Interesse, sich so zu verhalten, dass möglichst kein zweiter auftritt. Auch bei der Sekundärprävention spielen Bewegung und körperliche Aktivität eine große Rolle, generell Ernährung, Nichtrauchen und moderater Alkoholkonsum. Zur Sekundärprävention gehört auch die medikamentöse Therapie und, damit eng verbunden, die Therapietreue. Die Tertiärprävention schließlich bezeichnet alle Maßnahmen, mit denen chronisch kranke Patientinnen/Patienten das Fortschreiten der Erkrankung verlangsamen und/oder ihre Lebensqualität erhalten können. Die Erkrankung selbst kann damit zwar nicht verhindert oder geheilt werden, aber die Krankheitsfolgen können abgemildert werden. Beispiele aus dem Bereich der Neurologie sind die Multiple Sklerose, wo sich die Lebensführung auf den Krankheitsverlauf auswirken kann, oder die Parkinson-Erkrankung, bei der es gute Evidenz dafür gibt, dass Sport und gezielte Bewegung zu einer Symptomlinderung führt und die Mobilität erhält.
Die Kommission „Prävention in der Neurologie" möchte das Wissen zu diesen Bereichen zusammentragen, erweitern und dazu beitragen, dass neurologische Erkrankungen weniger häufig auftreten und bei Auftreten besser kontrolliert werden können. In der neuen Konstellation sind daher Mitglieder aus verschiedenen Spezialgebieten der Neurologie vertreten. Allerdings bleiben aufgrund der $\mathrm{Pa}$ tientenkontakte und der Versorgungsstrukturen die Aspekte der Sekundär- und Tertiärprävention wesentlich indikationsbezogen zugeordnet und auch in der Rehabilitations- und Sozialmedizin verankert. Aufgabe der Kommission „Prävention“ ist es, sich vorrangig mit grundlegenden primärpräventiven Maßnahmen auseinanderzusetzen. Sie will damit auch einen Beitrag leisten zu dem, was man heute „Public Health" nennt, generelle Gesundheitsvorsorge in der Bevölkerung.

Auf welche Herausforderungen in der Neurologie hat die DGN mit der Gründung der Kommission reagiert?

Die Kommission „Prävention in der Neurologie" ist aus der Kommission „Sport und Neurologie" hervorgegangen. Diese Kommission war, wie der Name bereits sagt, auf Sport und Bewegung ausgerichtet - und ihre Mitglieder waren bzw. sind sehr sportlich bzw. sportaffin. Die Kommission hat beispielsweise über Jahre beim DGN-Kongress einen Sponsorenlauf organisiert. Die DGN hat es nun für erforderlich erachtet, die Kommission noch breiter aufzustellen und auch die anderen Aspekte der Prävention wie Ernährung (und Nahrungsergänzung), Schlaf, Lebensführung abzudecken, auch wenn Bewegung, körperliche Aktivität und Sport nach wie vor ganz wesentliche Elemente darstellen.

Auf welche Herausforderungen die DGN damit reagiert hat, möchte ich einmal plakativ auf zwei Stichworte bringen: Alter und Volkskrankheiten. Die Gesellschaft wird immer älter und es sind gerade die Volkskrankheiten Schlaganfall und Demenz, die zu gravierenden Einbußen der Lebensqualität führen. Insofern spielt die Neurologie für die Gesundheit und ein „gutes Leben" bis ins hohe Alter eine große Rolle und sollte daher auch im Bereich der Prävention dieser Erkrankungen sichtbarer werden. Noch immer steht der Schlaganfall in der öffentlichen Wahrnehmung hinter dem Herzinfarkt und mit welchen Maßnahmen man z. B. einer Demenz entgegenwirken oder das Fortschreiten einer Parkinson-Krankheit verlangsamen kann, ist in der Bevölkerung wenig bekannt. Es ist meines Erachtens ganz wichtig, dass die Neurologie da mehr nach außen in Erscheinung tritt und wir uns neben den Kardiologen und Onkologen in dem Bereich Prävention positionieren und auch zusammenarbeiten. Denn viele Präventionsmaßnahmen beugen gleich mehreren Erkrankungen vor, also gleichzeitig Gefäßerkrankungen, degenerativen Erkrankungen und selbst Tumorerkrankungen.

Welche Meilensteine will die Kommission in den nächsten zwölfMonaten erreichen?

Zunächst möchte ich anmerken, dass unsere Kommission zwar auf dem DGN-Kongress 2019 konstituiert wurde, aber erst danach die Mitglieder berufen wurden. Bisher hatten die Kommissionsmitglieder noch nicht die Gelegenheit, sich zu einer ersten Sitzung zusammenzufinden, was auch der COVID-19-Pandemie geschuldet ist. Aber natürlich stehen wir bereits im Austausch und haben auch schon erste Ziele definiert. Wir möchten Präventionsthemen in die Fachgesellschaft hinein kommunizieren. Beim kommenden virtuellen Online-Kongress der DGN planen wir eine Sitzung mit dem Titel „Prävention neurologischer Erkrankungen - die Bedeutung von Bewegung, Ernährung, Kognition und Schlaf“.
Ein zweites konkretes Projekt ist, einen Übersichtsartikel zu erstellen, in dem wir die „harte Evidenz" von Prävention, vor allem im Bereich der Primärprävention von Schlaganfall und Demenz, zusammentragen wollen. Wir hoffen, einen solchen Artikel zum Ende des Jahres für die „DGNeurologie“ fertigstellen zu können.

Was sind längerfristige Ziele, die Sie ins Auge gefasst haben?

Nun, wir möchten perspektivisch mit anderen indikationsgebundenen Kommissionen (z. B. Kommission Zerebrovaskuläre Erkrankungen, MS etc.) zusammenarbeiten, um z.B. die Leitlinienarbeit um den Aspekt der Prävention zu erweitern bzw. auszubauen. Wir möchten vernetzen, Synergien ausloten und neue Formate auflegen, um die Prävention auch innerhalb des Fachs zu verankern.

Aber wir möchten auch nach außen strahlen, von besonderer Wichtigkeit ist die Sichtbarmachung und Verankerung der neurologischen Prävention in der Bevölkerung. Der Öffentlichkeitsarbeit kommt somit eine hohe Bedeutung zu. Auch hier gilt es, erfolgreiche Formate zu schaffen. Bei uns in Moers beispielsweise veranstalten wir jedes Jahr einen „Lauf gegen den Schlaganfall“, der inzwischen sehr viel öffentliches Interesse generiert. Laufen schützt vor Schlaganfall und Demenz, macht gelassener: Laufen ist Arbeiten am Ruhepuls! Da stellt sich die Frage, wie man solche Events bundesweit ausweiten kann, um die Prävention neurologischer Erkrankungen in den Fokus zu rücken. Ich hoffe da auch auf eine erfolgreiche Zusammenarbeit mit der Deutschen Hirnstiftung, die nächstes Jahr unter der Leitung von Prof. Dr. Frank Erbguth ihre Arbeit aufnehmen wird und neurologische Präventionsthemen in die Öffentlichkeit tragen wird; wir stehen bereits im Kontakt.

Interview: Dr. Bettina Albers 


\section{Ausschreibungen}

\author{
Aktuelle Ausschreibungen der DGN \\ und anderer Organisationen sowie \\ weitere Informationen finden Sie \\ unter: www.dgn.org/rubrik-dgn/ \\ ausschreibungen-und-preise
}

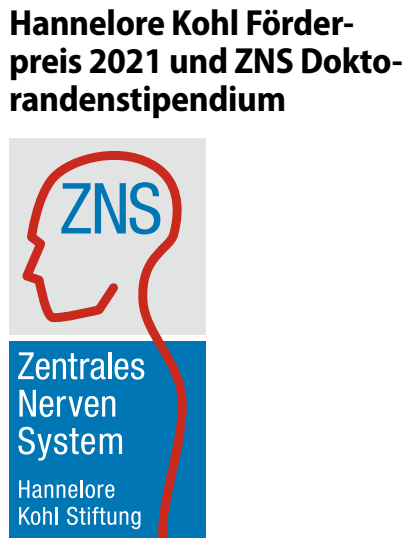

Die Förderung der neurowissenschaftlichen Forschung hat bei der ZNS - Hannelore Kohl Stiftung eine lange Tradition: Seit der Gründung wurden allein in diesem Bereich über 160 Projekte aus Spendenmitteln finanziert. Auch im Jahr 2020 fördert die Stiftung Projekte, die die Lebensqualität und die Heilungschancen von Menschen mit unfallbedingten Schädigungen des Gehirns verbessern oder im Rahmen der Prävention durch gezielte Aufklärungsarbeit über Unfallursachen und -risiken aufklären.

Der Hannelore Kohl Förderpreis ist mit 10.000 Euro dotiert und wird für eine Publikation von hoher wissenschaftlicher Qualität als Auszeichnung für hervorragende Leistungen des wissenschaftlichen Nachwuchses vergeben. Berücksichtigt werden Originalarbeiten aus den Bereichen der klinischen Forschung und Entwicklung mit direktem Bezug zum Thema Schädelhirntrauma. Publikationen dürfen bei der Einreichung nicht älter als zwei Jahre, weder schon anderweitig ausgezeichnet noch für eine Preisvergabe eingereicht worden sein.

Für das ZNS Doktorandenstipendium wird eine klinische, klinisch-experimentelle oder expe- rimentelle Doktorarbeit auf dem Gebiet der Neurowissenschaften mit dem Schwerpunkt Schädelhirntrauma gefördert. Für das Stipendium sind insgesamt 10.000 Euro vorgesehen, die Auszahlung erfolgt monatlich. Die Förderlaufzeit beträgt zwölf Monate. Beginn des Stipendiums ist der 1. April 2021.

Bewerbungen für beide Auszeichnungen sind bis zum 31. Oktober 2020 online einzureichen. Weitere Informationen: https://www.hannelore-kohl-stiftung.de

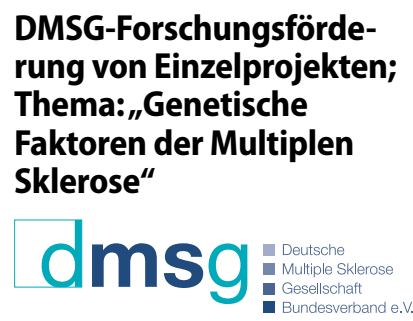

Der Bundesverband der Deutschen Multiple Sklerose Gesellschaft engagiert sich, zusammen mit Ärztinnen/Ärzten, Wissenschaftlern, Erkrankten, Angehörigen und allen Interessierten, dafür, die Erforschung der Multiplen Sklerose (MS) voranzutreiben. Dafür hat er eine Forschungsförderung ins Leben gerufen

Die Ursache der MS und die den Verlauf modifizierenden Einflüsse sind noch nicht geklärt. MS ist keine vererbbare Erkrankung entsprechend der klassischen Vererbungslehre, sondern verschiedene Genvarianten scheinen in der Summe das Risiko, also die Disposition, zu erhöhen. Nach aktuellem Kenntnisstand wird von einer Kombination aus etwa $30 \%$ genetischer Veranlagung und ca. 70 \% Risikofaktoren aus der Umwelt ausgegangen. Inwieweit die genetische Zusammensetzung Einfluss auf den Krankheitsverlauf hat, ist bisher größtenteils unbekannt. Im Rahmen der Ausschreibung sollen Forschungen zu genetischen Faktoren der MS gefördert werden, um einerseits unser Wissen über die Prädisposition zu erweitern und andererseits Erkenntnisse zu erhalten, ob und ggf. wie sich die genetische Ausstattung auf den Verlauf der MS auswirken kann.

Im jährlichen Ausschreibungsturnus werden exzellente Projekte aus angewandter oder klinischer Forschung zum jeweiligen Jahresthema (s.o.) für maximal 24 Monate gefördert. Das projektindividuelle Fördervolumen kann bis zu 100.000 Euro/ Projekt und Jahr betragen. Die Anzahl der geförderten Projekte und die Bewilligung einer Förderung sind abhängig von den für die Forschung zur Verfügung stehenden zweckgebundenen Mitteln und der Beschlussfassung durch den Geschäftsführenden Vorstand. Beginn der Förderung ist frühestens ab Mai 2021 möglich.

Ihre vollständigen Antragsunterlagen senden Sie bitte bis zum 30. November 2020 (Eingangsfrist) mit dem Betreff: „Einzelprojekt Forschungsförderung “ an referat-gs@dmsg.de

Weitere Informationen zum Antrag sowie dem unabhängigen Begutachtungsprozess entnehmen Sie bitte den Richtlinien zur Forschungsförderung der DMSG, Bundesverband e. V., und unserer Website unter www. dmsg.de/unsere-aufgaben/wirfoerdern-die-forschung/

\section{DGNI-Pflege- und Thera- piepreis 2020}

Die Deutsche Gesellschaft für NeuroIntensiv- und Notfallmedizin schreibt in diesem Jahr zum vierten Mal den DGNIPflege- und Therapiepreis aus. Mit diesem Preis möchte die Fachgesellschaft Pflegekräfte und TherapeutInnen würdigen, die mit ihrem professionellen pflegerischen und therapeutischen Wissen und Handeln zur Verbesserung der Versorgung von Neurointensivpatientinnen /-patienten beitragen. Der Preis ist mit 500 Euro dotiert. Eingereicht werden können veröf- fentlichte oder unveröffentlichte Texte (Abschlussarbeiten, Projektberichte, Studien, Innovationen etc.) in deutscher Sprache. Bitte senden Sie Ihren Beitrag als PDF per E-Mail bis zum 30. November 2020 an Peter Nydahl: Peter.Nydahl@uksh.de. Weitere Informationen finden Sie unter https://www.dgni.de/

\section{Wissenschaftspreis der Deutschen Gesellschaft für Liquordiagnostik und Klinische Neurochemie e.V. (DGLN)}

Der Preis wird von der Deutschen Gesellschaft für Liquordiagnostik und Klinische Neurochemie e. V. ausgeschrieben. Prämiert werden deutschsprachige klinisch-neurologisch und klinisch-chemisch tätige Wissenschaftlerinnen/Wissenschaftler für ein abgeschlossenes wissenschaftliches Projekt zum Thema Liquordiagnostik, das in den Jahren 2019 und 2020 zur Publikation eingereicht oder angenommen bzw. in den letzten 24 Monaten vor Ablauf der Bewerbungsfrist publiziert wurde.

Der Preis wird anlässlich der alle zwei Jahre stattfindenden Jahrestagung der DGLN in Form einer Urkunde überreicht und ist mit einer Geldprämie von 2000 Euro verbunden. Die Preisverleihung zur aktuellen Ausschreibung findet bei der Jahrestagung der DGLN in Erlangen (07.05.-08.05.2021) statt.

Den Unterlagen sind das Manuskript bzw. die Publikation, das Schriftenverzeichnis und der Lebenslauf beizulegen. Sie sind als PDF per E-Mail bis zum 31. Dezember 2020 zu richten an die Geschäftsstelle der DGLN e.V.: info@dgln.de 


\section{Save the Date}

Bitte informieren Sie sich, ob die aufgeführten Veranstaltungen wegen der aktuellen Einschränkungen durch die SARS-CoV-2-Pandemie wie geplant stattfinden können!

\section{Fortbildungen}

Würzburger Elektrophysiologie Workshop, 16. Oktober 2020, Würzburg

Curriculärer Herbst- und Frühjahrskurs Epileptologie 2020/2021 Kursteil 1, 31. Oktober 2020, Bochum

15. Bamberger Neuro-Psychiatrie-Symposium, 14. November 2020, Bamberg

Refresher-Kurs - Spezielle Neurologische Ultraschalldiagnostik, 7. Dezember 2020, Essen

\section{Online-Fortbildung}

ERN-RND-Webinare zu seltenen Bewegungsstörungen und neuromuskulären Erkrankungen

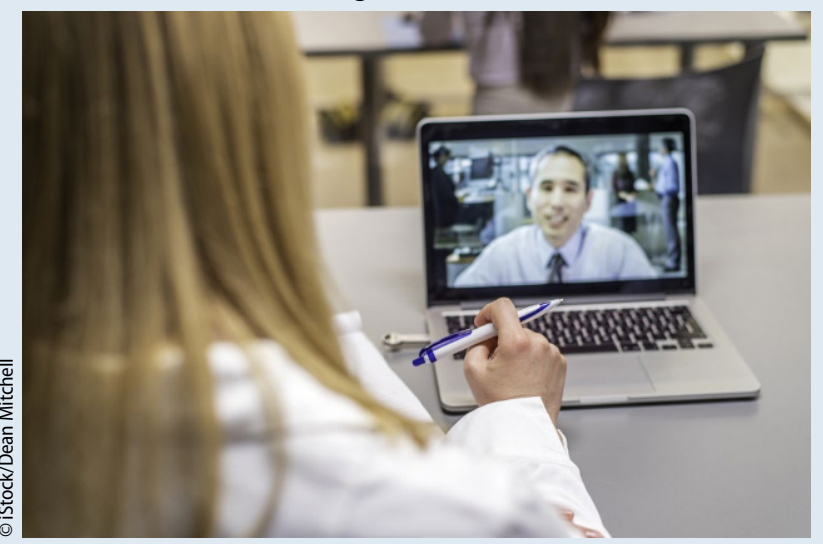

Um Neurologen europaweit im Bereich seltenerer neurologischer Bewegungsstörungen und neuromuskulärer Erkrankungen zu schulen, bietet die ERN-RND Webinare unter der Leitung europäischer Experten an.

Die Webinare wurden in Zusammenarbeit mit dem „European Reference Network for Rare Neuromuscular Diseases" (EURO-NMD) und der "European Academy of Neurology“ (EAN) aufgelegt und werden von verschiedenen europäischen Experten durchgeführt. Es werden sowohl Erkrankungen des Kindes- und Jugendalters als auch des Erwachsenenalters thematisiert.

Eine Übersicht aller Termine finden Sie hier: http://www.ern-rnd.eu/ education-training/webinars

\section{FEES-Expertenworkshops}

26.-27. Oktober 2020, München Weitere Informationen online: www.dgn.org/rubrik-themen/fees

\section{Kongresse und Symposien}

28. Jahrestagung der Deutschen Gesellschaft für Schlafforschung und Schlafmedizin DGSM, 29. Oktober 2020, Essen, Digitaler Kongress, https://www.dgsmkongress.de

28. Jahrestagung der Gesellschaft für Schädelbasischirurgie (GSB) e. V., verschoben auf Herbst 2021, München, www.gsb-kongress.de

93. Kongress der Deutschen Gesellschaft für Neurologie, 4.-7. November 2020, Berlin, Digitaler Kongress, www.dgnkongress.org

15. Jahrestagung Deutsche Gesellschaft Interdisziplinäre Notfall- und Akutmedizin (DGINA) e. V., 11.-13. November 2020, Wolfsburg, Digitaler Kongress, www.dgina-kongress.de

5. Mitteldeutsches Neuroradiologie-Symposium (Präsenz \& Virtuell), 12.-14. November 2020, Leipzig, www.mitteldeutscheneuroradiologie.de

\section{Personalia}

Haben Sie eine Leitungsposition übernommen oder eine Praxis gegründet? Wollen Sie, dass Ihre Kolleginnen und Kollegen von Ihrer beruflichen Veränderung erfahren? Dann informieren Sie die Geschäftsstelle der DGN unter der E-Mail-Adresse: info@dgn.org.

\section{Ehrung}

Prof. Dr. Wilfred Nix, ehem. Leitender Oberarzt der Klinik für Neurologie der Universitätsmedizin Mainz wurde von der Bundesärztekammer die Ernst-
III. International Conference on Deep Brain Stimulation, 20.21. November 2020, Digitaler Kongress, www.dbs-conference.com

46. Jahrestagung der Sektion Intrakranieller Druck, Hirndurchblutung und Hydrozephalus der Deutschen Gesellschaft für Neurochirurgie (DGNC) e. V., 20.21. November 2020, Heidelberg, www.sektionstagung-icp.de

20. Kongress der Deutschen Interdisziplinären Vereinigung für Intensiv- und Notfallmedizin e. V., 2.-4. Dezember 2020, Digitaler Kongress, www.divi20.de

15. Deutscher Wirbelsäulenkongress, 10.-12. Dezember 2020, Berlin, Digitaler Kongress, www. dwg-kongress.de

8. Gemeinsame Jahrestagung der Deutschen Gesellschaft für Neurorehabilitation DGNR und der Deutschen Gesellschaft für Neurotraumatologie und Klinische Neurorehabilitation DGNKN, 10.-12. Dezember 2020, Digitaler Kongress, www. dgnr-dgnkn-tagung.de

Weitere Informationen online: www.dgn.org/fortbildungskalender

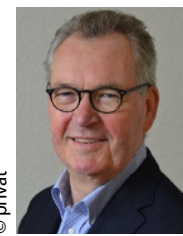

vo n - B e r g mann-Plakette für seine Verdienste um die ärztliche Fortbildung verliehen. 22 Jahre lang leitete er, neben seiner Tätigkeit in der Universitätsmedizin, die Akademie für Ärztliche Fortbildung Rheinland-Pfalz. Er ist seit dem 01.01.1983 DGNMitglied. 


\section{In den Ruhestand verab- schiedet}

Jörg-Henning Spelmeyer, vorher Oberarzt im Evangelischen

\section{DGNv Neurojobs}

\section{Aktuelle Jobangebote}

\section{Chefarztpositionen}

46242 Knappschaftskrankenhaus Bottrop, Chefarzt für Neurologie (m/w/d)

78262 Klinik in Gailingen am Hochrhein (Kliniken Schmieder), Chefarzt für die Neurologische Rehabilitation $(\mathrm{m} / \mathrm{w} / \mathrm{d})$

Schweiz:

8008 Klinik Lengg, Zürich (CH), Medizinischer Direktor Epilepto$\operatorname{logie}(\mathrm{m} / \mathrm{w} / \mathrm{d})$

Österreich:

4400 Pyhrn-Eisenwurzen Klinikum Steyr (A), Chefarzt für Neurologie $(\mathrm{m} / \mathrm{w} / \mathrm{d})$

\section{Oberarztpositionen}

01896 Klinik Schloss Pulsnitz, Oberarzt (m/w/d)

19395 Mediclin Krankenhaus Plau am See, Oberarzt (m/w/d) 27574 Klinikum BremerhavenReinkenheide, Ltd. Oberarzt (m/w/d)

29525 Helios Klinikum Uelzen, Oberarzt Neurologie (m/w/d) 41812 Hermann-Josef-Krankenhaus Erkelenz, Oberarzt (m/w/d) 44309 Knappschaftskrankenhaus, Dortmund, Oberarzt (m/w/d) 49152 Dr. Becker Neurozentrum Niedersachsen, Bad Essen, Ober$\operatorname{arzt}(\mathrm{m} / \mathrm{w} / \mathrm{d})$

59755 Klinikum Hochsauerland, St. Johannes-Hospital, ArnsbergNeheim, Oberarzt $(\mathrm{m} / \mathrm{w} / \mathrm{d})$ 63225 Asklepios Klinik Langen, Oberarzt $(\mathrm{m} / \mathrm{w} / \mathrm{d})$

63739 Klinikum Aschaffenburg-Alzenau gGmbH, Oberarzt $(\mathrm{m} / \mathrm{w} / \mathrm{d})$

70376 Robert-Bosch-Krankenhaus Stuttgart, Oberarzt $(\mathrm{m} / \mathrm{w} / \mathrm{d})$ 71640 Klinikum Ludwigsburg, Oberarzt $(\mathrm{m} / \mathrm{w} / \mathrm{d})$
Krankenhaus Hattingen gGmbH geht zum 01.10.2020 in den $\mathrm{Ru}-$ hestand. Er ist seit 01.01.1996 DGN-Mitglied.

72488 SRH Krankenhaus Sigmaringen, Oberarzt $(\mathrm{m} / \mathrm{w} / \mathrm{d})$ 74206 SRH Gesundheitszentrum Bad Wimpfen, Oberarzt (m/w/d) 78262 Hegau Jugendwerk Gailingen, Oberarzt für Neuropädiatrie (m/w/d)

80804 Schön Klinik München Schwabing, Oberarzt $(\mathrm{m} / \mathrm{w} / \mathrm{d})$ 87600 Bezirkskliniken Schwaben Kaufbeuren, Oberarzt (m/w/d) 91056 Bezirkskliniken Mittelfranken - Erlangen, Oberarzt (m/w/d)

\section{Facharztpositionen}

01896 Klinik Schloss Pulsnitz, Facharzt Neurologie (m/w/d) 06112 BG Klinikum Halle, Facharzt Neurologie $(\mathrm{m} / \mathrm{w} / \mathrm{d})$

14770 Städt. Klinikum Brandenburg, Facharzt Neurologie $(\mathrm{m} / \mathrm{w} / \mathrm{d})$

22087 Marienkrankenhaus Hamburg, Facharzt Neurologie $(\mathrm{m} / \mathrm{w} / \mathrm{d})$

24159 Praxis Sospesa PFADEN

- Kiel, Facharzt Neurologie $(\mathrm{m} / \mathrm{w} / \mathrm{d})$

37075 Universitätsmedizin Göttingen, Facharzt Neurologie $(\mathrm{m} / \mathrm{w} / \mathrm{d})$

40670 St. Mauritius Therapieklinik Meerbusch Düsseldorf, Facharzt Neurologie $(\mathrm{m} / \mathrm{w} / \mathrm{d})$ 44309 Knappschaftskrankenhaus Dortmund, Facharzt Neurologie $(\mathrm{m} / \mathrm{w} / \mathrm{d})$

49152 Dr. Becker Neurozentrum Niedersachsen, Bad Essen, Facharzt Neurologie $(\mathrm{m} / \mathrm{w} / \mathrm{d})$ 67655 Westpfalz-Klinikum Kaiserslautern, Facharzt Neurologie $(\mathrm{m} / \mathrm{w} / \mathrm{d})$

71065 Kreiskliniken Böblingen Sindelfingen, Facharzt Neurologie $(\mathrm{m} / \mathrm{w} / \mathrm{d})$
71364 Rems-Murr Kliniken Winnenden, Facharzt Neurologie $(\mathrm{m} / \mathrm{w} / \mathrm{d})$

78262 Hegau Jugendwerk Gailingen, Facharzt Neurologie (m/w/d) 80804 Schön Klinik München Schwabing, Facharzt Neurologie (m/w/d)

83043 Schön Klinik Bad Aibling Harthausen, Facharzt Neurologie $(\mathrm{m} / \mathrm{w} / \mathrm{d})$

83646 CoMedicum Bad Tölz, Facharzt Neurologie (m/w/d) 89081 Universitätsklinikum Ulm, Facharzt Neurologie (m/w/d) 96047 Facharztzentrum Löwenbrücke Bamberg, Facharzt Neurologie $(\mathrm{m} / \mathrm{w} / \mathrm{d})$

Schweiz:

3012 Swissmedic - Bern $(\mathrm{CH})$, Clinical Reviewer 80-100\% $(\mathrm{m} / \mathrm{w} / \mathrm{d})$

\section{Ärzte in Weiterbildung - Positionen für Assistenzärzte $(\mathrm{m} / \mathrm{w} / \mathrm{d})$ in der Neurologie} 04552 Sana Klinikum Leipziger Land, Borna

06886 Alexianer Klinik Bosse Wittenberg

06112 BG Klinikum Halle

13509 Vivantes Humboldt-Klinikum Berlin

13585 Vivantes Klinikum BerlinSpandau

14770 Städt. Klinikum Brandenburg

22087 Marienkrankenhaus Ham-

burg

22307 Asklepios Klinik Barmbek - Hamburg

22417 Asklepios Klinik Nord Hamburg

23562 UKSH Campus Lübeck

24159 Praxis Sospesa PFADEN

- Kiel

24768 imland Klinik Rendsburg

27574 Klinikum Bremerhaven-

Reinkenheide

30625 Medizinische Hochschule

Hannover

37075 Universitätsmedizin Göttingen

39120 Universitätsmedizin Magdeburg 39130 Klinikum Magdeburg 40479 Marienhospital Düsseldorf 40670 St. Mauritius Therapieklinik Meerbusch Düsseldorf 42697 St. Lukas Klinik Solingen
44309 Knappschaftskrankenhaus Dortmund

45964 St. Barbara Hospital in Gladbeck

46325 St. Marien-Hospital Borken 47906 Hospital zum Heiligen Geist Kempen

49152 Dr. Becker Neurozentrum Niedersachsen, Bad Essen 58515 Klinikum Lüdenscheid 65929 Klinikum Frankfurt Höchst 66424 Universitätsklinikum des Saarlandes (UKS) Homburg 66740 DRK-Krankenhaus Saarlouis

67655 Westpfalz Klinikum Kaiserslautern

68167 Universitätsmedizin Mannheim

71364 Rems-Murr Kliniken Winnenden

77933 Ortenau Klinikum LahrEttenheim

79539 Kliniken des Landkreises

Lörrach

80804 Schön Klinik München Schwabing 83022 RoMed Klinikum Rosenheim

83043 Schön Klinik Bad Aibling Harthausen

86156 Universitätsklinikum Augsburg

87600 Bezirkskliniken Schwaben

- Kaufbeuren

88212 Oberschwabenklinik St. Elisabethen-Klinikum Ravensburg

89081 Universitätsklinikum Ulm

97422 Leopoldina Krankenhaus Schweinfurt

99891 MEDICLIN Klinik am Rennsteig Bad Tabarz

Kliniken Schmieder: Standorte: Gailingen, Allensbach, Konstanz Stuttgart-Gerlingen, Heidelberg

Luxemburg

1445 Luxembourg Institute of Health (L), Medical Doctors-PhD and $\mathrm{PhD}$-Students $(\mathrm{m} / \mathrm{w} / \mathrm{d})$

(Stand 26.08.2020)

Weitere Jobangebote finden Sie tagesaktuell online auf www. dgn-neurojobs.org 